\title{
Rationality criteria for motivic zeta functions
}

\author{
Michael Larsen and Valery A. Lunts
}

\begin{abstract}
The zeta function of a complex variety is a power series whose $n$th coefficient is the $n$th symmetric power of the variety, viewed as an element in the Grothendieck ring of complex varieties. We prove that the zeta function of a surface is rational if and only if the Kodaira dimension of the surface is negative.
\end{abstract}

\section{Introduction}

Let $X$ be a variety over a finite field $\mathbb{F}$ and $\operatorname{Sym}^{n} X=X^{n} / S_{n}$, the variety of effective 0 -cycles on $X$ of degree $n$ (where, by convention, $\operatorname{Sym}^{0} X=\operatorname{Spec} \mathbb{F}$ ). A celebrated theorem of B. Dwork asserts that the zeta function

$$
Z_{X}(t)=\sum_{i=0}^{\infty}\left|\operatorname{Sym}^{n}(X)(\mathbb{F})\right| t^{n}
$$

is a rational function in $t$. Kapranov asked [Kap00] whether this rationality lifts to the Grothendieck ring of varieties over $\mathbb{F}$. Explicitly, let $K_{0}\left[\mathcal{V}_{\mathbb{F}}\right]$ denote the ring of $\mathbb{Z}$-combinations of isomorphism classes of $\mathbb{F}$-varieties modulo the cutting-and-pasting relation

$$
[X]=[Y]+[X \backslash Y]
$$

for closed $\mathbb{F}$-subvarieties $Y \subset X$. Is the motivic zeta function

$$
\zeta_{X}(t)=\sum_{i=0}^{\infty}\left[\operatorname{Sym}^{n}(X)\right] t^{n}
$$

always rational as a power series in $K_{0}\left[\mathcal{V}_{\mathbb{F}}\right]$ ? More generally, is this true for varieties over a general field $K$, for example, $K=\mathbb{C}$ ? If so, this would give a fundamentally new proof of Dwork's theorem, one that does not depend on Frobenius at all. Kapranov observed that it is true when $X$ is a curve. (See Theorem 3.7 below for a statement and proof over $\mathbb{C}$.)

In [LL03], we proved that $\zeta_{X}(t)$ is not rational when $X$ is a complex projective non-singular surface with geometric genus $\geqslant 2$. We should point out that Kapranov's set-up is slightly different from ours, in that he inverts $\left[\mathbb{A}^{1}\right]$ whereas we do not. In principle, it is still possible that $\zeta_{X}(t)$ is always rational as a power series over $K_{0}\left[\mathcal{V}_{\mathbb{C}}\right]\left[\left[\mathbb{A}^{1}\right]^{-1}\right]$. This paper does not address that interesting possibility. Instead, we return to the problem of [LL03] and give a simple necessary and sufficient condition on complex surfaces for $\zeta_{X}(t)$ to be rational over $K_{0}\left[\mathcal{V}_{\mathbb{C}}\right]$, as follows.

Theorem 1.1. A non-singular projective complex surface has rational motivic zeta function if and only if it has Kodaira dimension $-\infty$.

Received 7 January 2003, accepted in final form 13 October 2003, published online 15 October 2004. 2000 Mathematics Subject Classification 14F42, $14 \mathrm{G} 10$.

Keywords: Grothendieck group, motivic zeta function, motivic measure, $\lambda$-ring, algebraic surface, Dwork's theorem.

The first named author was partially supported by NSF grant DMS-0100537. The second named author was partially supported by NSA grant MDA904-01-1-0020 and CRDF grant RM1-2405-MO-02.

This journal is (C) Foundation Compositio Mathematica 2004. 


\section{LARSEn AND V. A. Lunts}

The notion of rationality needs some explanation (see $\S 2$ below). Theorem 3.9 asserts rationality in the strong sense when the Kodaira dimension is $-\infty$, and Theorem 7.6 denies rationality in the weak sense when the Kodaira dimension is $\geqslant 0$. The methods for proving rationality and irrationality are entirely different. The fact that they meet in the middle to give a necessary and sufficient condition appears as a minor miracle, made possible by the classification of surfaces [Rei97, E.1]. By contrast, in dimensions $\geqslant 3$, the rationality problem seems wide open.

There are two main ideas in this paper. The first is to probe rationality by means of new motivic measures, i.e. field-valued points of Spec $K_{0}\left[\mathcal{V}_{\mathbb{C}}\right]$. In [LL03], we showed that any multiplicative function on the set of stable birational equivalence classes of non-singular complex projective varieties defines a motivic measure. In particular, we constructed a measure $\mu_{1}$, with values in the fraction field of the group ring of the multiplicative group of integer power series $1+s \mathbb{Z}[[s]]$, characterized by the formula

$$
\mu_{1}([Z])=\left[\sum_{i=0}^{\operatorname{dim} Z} \operatorname{dim} \Gamma\left(Z, \Omega_{Z}^{i}\right) s^{i}\right]
$$

for $Z$ a (connected) non-singular projective variety. In the current paper, we introduce a set of measures $\mu_{n}$ indexed by positive integers $n$, characterized by

$$
\mu_{n}([Z])=\left[\sum_{i=0}^{\operatorname{dim} Z} \operatorname{dim} H^{0}\left(Z, \Psi^{n} \Omega_{Z}^{i}\right) s^{i}\right],
$$

where $\Psi^{n}$ denotes the $n$th Adams operation. The point of this generalization is that it allows us to work with higher plurigenera in much the way that we worked with geometric genus in [LL03]. If for some $n, \mu_{n}\left(\zeta_{X}(t)\right)$ is irrational, then of course $\zeta_{X}(t)$ itself is irrational. For singular $Z$, the right-hand side of (1) does not make sense, and indeed the left-hand side is generally not of the form $[P]$ for any power series $P \in 1+s \mathbb{Z}[[s]]$. However, in the special case $Z=\operatorname{Sym}^{m} X$, $\operatorname{dim} X=2$, it turns out that $\mu_{n}([Z])=\mu_{n}\left(\left[\right.\right.$ Hilb $\left.\left.^{m} X\right]\right)$, where Hilb ${ }^{m} X$ is the Hilbert scheme of length $m$ subschemes of $X$. (The fact that Hilb ${ }^{m} X$ is non-singular is another way in which dimension 2 is special.) Our task, therefore, is to prove that if the $n$th plurigenus of $X$ is positive, then

$$
\sum_{i=0}^{\infty} \mu_{n}\left(\left[\operatorname{Hilb}^{i} X\right]\right) t^{i}
$$

is irrational.

The other main idea in this paper is to make systematic use of $\lambda$-ring ideas and techniques (see $\S 4$ for a brief review of this theory). We have already noted the appearance of Adams operations. This is somewhat delicate: we cannot work in the usual $K(X)$ since the global section functor is not well defined there. Instead, we need to prove that the ring of virtual vector bundles modulo split exact sequences is a special $\lambda$-ring (see $\S 5$ ). Also significant is the idea that the motivic zeta function (or better, its inverse) should be regarded as the universal $\lambda$-homomorphism. This is true only formally since $K_{0}\left[\mathcal{V}_{\mathbb{C}}\right]$ is not a special $\lambda$-ring. It is nonetheless suggestive, since the universal $\lambda$-homomorphism sends every virtually finite element to a rational power series. For a special $\lambda$-ring the virtually finite elements form a $\lambda$-subring, which in many interesting cases is the whole ring. This interpretation of the zeta function suggests on the one hand that we should seek motivic measures which are $\lambda$-homomorphisms and on the other that it may be natural to replace the Grothendieck ring of varieties by its specialization, i.e. its maximal quotient, which is a special $\lambda$-ring (see $\S 8$ ).

E. Looijenga has called our attention to certain formal analogies between the problem of rationality of zeta functions of complex surfaces $X$, and Severi's conjecture, disproved by Mumford [Mum68], which predicted that the group of 0 -cycles modulo rational equivalence on $X$ would be finite-dimensional. Each statement can be understood as bounding the complexity of 


\section{RATIONALITY CRITERIA FOR MOTIVIC ZETA FUNCTIONS}

symmetric powers of $X$. In each case, global holomorphic 2-forms provide the obstruction to this boundedness. However, there is an essential difference. A conjecture of Bloch [Blo75] asserts that, when $H^{0}\left(X, \Omega_{X}^{2}\right)=0$, the group of degree-zero 0-cycles should be isomorphic to the Albanese variety of $X$. This has been settled by Bloch et al. [BKL76] for Kodaira dimension $<2$, so there are examples of surfaces in which the two notions of boundedness diverge. In particular, the use of plurigenera in this paper does not seem to have a counterpart in the world of $\mathrm{CH}^{2}$.

Throughout this paper, a variety will be a reduced separated scheme of finite type over $\mathbb{C}$. The class $[X] \in K_{0}\left[\mathcal{V}_{\mathbb{C}}\right]$ of a variety $X$ will sometimes be written without brackets when it seems unlikely to lead to any confusion.

\section{Rationality criteria for power series}

In this paper we will be concerned with the rationality of power series with coefficients in a commutative ring $A$. It is not entirely clear how such rationality should be defined when $A$ is not an integral domain (and we know [Poo02] that the Grothendieck ring of varieties has zero-divisors). We consider several possible definitions.

Definition 2.1. A power series $f(t) \in A[[t]]$ is globally rational if and only if there exist polynomials $g(t), h(t) \in A[t]$ such that $f(t)$ is the unique solution of $g(t) x=h(t)$.

Definition 2.2. A power series $f(t)=\sum_{i} a_{i} t^{i} \in A[[t]]$ is determinantally rational if and only if there exist non-negative integers $m$ and $n$ such that

$$
D_{i m}=\operatorname{det}\left(\begin{array}{cccc}
a_{i} & a_{i+1} & \cdots & a_{i+m} \\
a_{i+1} & a_{i+2} & \cdots & a_{i+m+1} \\
\vdots & \vdots & \ddots & \vdots \\
a_{i+m} & a_{i+m+1} & \cdots & a_{i+2 m}
\end{array}\right)=0
$$

for all $i>n$.

It is classical [Bor94] that Definition 2.1 is equivalent to Definition 2.2 when $A$ is a field; in fact, we can take $n=\max (0, \operatorname{deg} h-\operatorname{deg} g)$ and $m=\operatorname{deg} g$. The easy direction, which works for any $\operatorname{ring} A$, is proved as part of Proposition 2.4 below. The difficult direction is proving that $D_{i m}=0$ for fixed $m$ and all $i \gg 0$ implies rationality. The idea of the proof is that, if $m$ is chosen to be minimal, then by induction $D_{i m-1} \neq 0$ for $i \gg 0$. This implies that, for $i \gg 0$, the row $\left(a_{i+m}, a_{i+m+1}, \ldots, a_{i+2 m}\right)$ is a linear combination of the rows $\left(a_{i+j}, a_{i+j+1}, \ldots, a_{i+j+m}\right)$ for $0 \leqslant j<m$, and, therefore, any linear relation satisfied by the coefficients of all rows but the last will also be satisfied for the coefficients of the last row. In particular, this enables us to prove that the coefficients of $a_{i}$ satisfy a fixed linear recurrence, and that implies global rationality of the power series $\sum_{i=0}^{\infty} a_{i} t^{i}$.

This equivalence suggests a third possible definition.

Definition 2.3. A power series $f \in A[[t]]$ is pointwise rational if and only if for all homomorphisms $\Phi$ from $A$ to a field, $\Phi(f)$ is rational by either of the two previous definitions.

These definitions are related by the following proposition.

Proposition 2.4. Any globally rational power series is determinantally rational, and any determinantally rational power series is pointwise rational. Neither converse holds for a general coefficient ring $A$. All three conditions are equivalent when $A$ is an integral domain.

Proof. Suppose $g(t)=\sum_{i=0}^{k} b_{i} t^{i}, h(t)=\sum_{i=0}^{\ell} c_{i} t^{i} \in A[[t]]$ and $f(t)=\sum_{i=0}^{\infty} a_{i} t^{i}$ is the unique solution to $g(t) x=h(t)$. Since $f(t)+a$ is not a solution when $a \neq 0$ is a constant, the annihilator 


\section{LARSEn And V. A. Lunts}

of the ideal $\left(b_{0}, b_{1}, \ldots, b_{k}\right)$ of coefficients of $g$ is $(0)$. Setting $m=k$, and $n=\max (0, \ell-k)$ in Definition 2.2,

$$
\left(\begin{array}{cccc}
a_{i} & a_{i+1} & \cdots & a_{i+m} \\
a_{i+1} & a_{i+2} & \cdots & a_{i+m+1} \\
\vdots & \vdots & \ddots & \vdots \\
a_{i+m} & a_{i+m+1} & \cdots & a_{i+2 m}
\end{array}\right)\left(\begin{array}{c}
b_{k} \\
b_{k-1} \\
\vdots \\
b_{0}
\end{array}\right)=\left(\begin{array}{c}
0 \\
0 \\
\vdots \\
0
\end{array}\right)
$$

for $i>\ell-k$. Left-multiplying both sides by the matrix of cofactors, we conclude that the determinant of the above matrix annihilates the $b$-column vector, which means that it is zero.

To see that the converse does not hold, consider $A=\mathbb{Z}[\epsilon] /\left(\epsilon^{2}\right)$. As $A$ is countable, the set of globally rational power series over $A$ is countable. However, any power series of the form $f(t)=\epsilon g(t)$ is determinantally rational in the sense of Definition 2.2 when $m=1$.

Suppose $f(t)=\sum_{i=0}^{\infty} a_{i} t^{i}$ is determinantally rational. The same is then true for $\Phi(f)$, which is a power series over a field. For fields, however, the determinantal condition implies rationality.

To see that the converse does not hold, consider

$$
A=\mathbb{Z}\left[\epsilon_{1}, \epsilon_{2}, \ldots\right] /\left(\epsilon_{1}^{2}, \epsilon_{2}^{2}, \ldots\right)
$$

in infinitely many variables $\epsilon_{i}$. Every homomorphism from $A$ to a field factors through the augmentation. Therefore, $f(t)=\sum_{i=1}^{\infty} \epsilon_{i} t^{i}$ is pointwise rational. However, the determinant

$$
\operatorname{det}\left(\begin{array}{cccc}
\epsilon_{i} & \epsilon_{i+1} & \cdots & \epsilon_{i+m} \\
\epsilon_{i+1} & \epsilon_{i+2} & \cdots & \epsilon_{i+m+1} \\
\vdots & \vdots & \ddots & \vdots \\
\epsilon_{i+m} & \epsilon_{i+m+1} & \cdots & \epsilon_{i+2 m}
\end{array}\right)
$$

is never zero since it has a non-trivial $\epsilon_{i} \epsilon_{i+2} \epsilon_{i+4} \cdots \epsilon_{i+2 m}$ coefficient.

Finally, when $A$ is an integral domain, we let $\Phi$ denote the inclusion map from $A$ to its fraction field $F$. If $f(t)$ is pointwise rational, it must be rational as a power series in $F[[t]]$. Therefore, $g(t) f(t)=h(t)$ for polynomials $g(t), h(t) \in F[t], g(t) \neq 0$. Clearing denominators, we may assume $g(t), h(t) \in A[t]$. As $A[t]$ is an integral domain, $f(t)$ is globally rational.

The following lemma will be useful in $\S 3$.

Lemma 2.5. If $f(t) \in 1+t A[[t]]$ is globally (respectively pointwise) rational, the same is true of $f(t)^{-1}$.

Proof. If $f(t)$ is the unique solution of $g(t) x=h(t)$, then $g(t)$ is not a zero-divisor in $A[[t]]$, and $f(t)$ is invertible, so again not a zero-divisor. Therefore $h(t)=f(t) g(t)$ is not a zero-divisor, and $f(t)^{-1}$ is the unique solution of $h(t) x=g(t)$. The pointwise case is trivial.

In [Kap00], the rationality of motivic zeta functions is discussed in pointwise terms. To give the strongest possible results, we generally prove rationality globally and irrationality pointwise. To do the latter, we make free use of the determinantal formulation of rationality for power series over fields.

The remainder of this section is devoted to a necessary and sufficient condition for rationality for power series of a very special type.

Consider a free abelian group $G$ and its group ring $\mathbb{Z}[G]$. This ring is isomorphic to a ring of Laurent polynomials and is therefore a domain. Let $F$ be its field of fractions. We are interested in power series in $F$ all of whose coefficients are of the form $[g], g \in G$. By a slight abuse of notation, we will denote such coefficients $g$. 


\section{RATIONALITY CRITERIA FOR MOTIVIC ZETA FUNCTIONS}

Definition 2.6. Let $K \subset G$ be a finite subset. We say that $h$ lies in the $K$-neighborhood of $g$ if and only if $h \in g K$.

Definition 2.7. Let $N$ be a non-negative integer. We say that a set $S$ of non-negative integers lies in an $N$-interval if there exists $a \in \mathbb{N}$ such that $S \subset[a, a+N]$.

DEFINITION 2.8. Let $r$ be a positive integer. We say a sequence $g_{0}, g_{1}, g_{2}, \ldots$ in $G$ has dispersion $\geqslant r$ if there exists an integer $N$ such that for every finite set $K \subset G$ there exists a set $S$ of $r$ integers contained in an $N$-interval such that, whenever $s$ and $t$ are distinct elements of $S, g_{s}$ does not lie in the $K$-neighborhood of $g_{t}$. Otherwise, it has dispersion $\leqslant r-1$. If a sequence has dispersion $\geqslant r$ and $\leqslant r$, we say it has dispersion $r$. We say a power series in $F[[t]]$ has given dispersion $(\geqslant r, \leqslant r$, or $=r$ ) if all of its coefficients are elements of $G$ and the sequence of its coefficients has the specified dispersion.

We note that, if a sequence has dispersion $\geqslant r$, we can take all the elements of $S$ to be arbitrarily large. Likewise, we can choose $N$ as large as we wish. If a sequence has dispersion $\leqslant r-1$, for all integers $N$ there exists a finite set $K$ such that every $r$-element set contained in an $N$-interval contains distinct elements $s, t$, with $g_{s}$ in the $K$-neighborhood of $g_{t}$. The sets $K$ can be enlarged freely.

Theorem 2.9. Let $f(t)=\sum g_{i} t^{i}$ be a power series in $F[[t]]$ with $g_{i} \in G$ for all $i$. Then $f(t)$ is rational if and only if there exists $p \geqslant 1, i_{0} \in \mathbb{N}$ and a sequence $h_{1}, h_{2}, h_{3}, \ldots \in G$ periodic with period $p$ such that for $i>i_{0}$ we have $g_{i+p}=h_{i} g_{i}$.

Proof. If there exist $p, i_{0}$, and $h_{i}$ as above, then

$$
f(t)=\sum_{i=0}^{i_{0}} g_{i} t^{i}+\sum_{i=1}^{p} \frac{g_{i_{0}+i} t^{i_{0}+i}}{1-h_{i+i_{0}} t^{p}}
$$

is a rational function with denominator of degree $m \leqslant p^{2}$.

For the converse, by assumption there exists a polynomial

$$
q(t)=b_{m} t^{m}+b_{m-1} t^{m-1}+\cdots+b_{0} \in F[t]
$$

such that $q(t) f(t)$ is a polynomial, whose degree we will denote $d$. Without loss of generality, we may assume $b_{0} \neq 0$ and $b_{m} \neq 0$, and clearing denominators, we may assume $b_{i} \in \mathbb{Z}[G]$ for all $i$. Let $C \subset G$ denote the collection of elements of the form $x y^{-1}$ where $x, y$ are group elements that have non-zero coefficient in some $b_{j}, 0 \leqslant j \leqslant m$. Thus, $C$ contains the identity and is closed under inverse. For $i>\max (0, d-m)$,

$$
b_{0} g_{i+m}+b_{1} g_{i+m-1}+\cdots+b_{m} g_{i}=0 .
$$

Expanding the left-hand side, every non-zero monomial appearing in a term $b_{j} g_{i+m-j}$, for some $j$ between 0 and $m$ inclusive, must also appear for at least one other value of $j$ in the interval. Applying this when $j=0$, we deduce

$$
g_{i+m} \in g_{i} C \cup \cdots \cup g_{i+m-1} C .
$$

In other words, $g_{i+m}$ belongs to the $C$-neighborhood of some element of the set $\left\{g_{i}, \ldots, g_{i+m-1}\right\}$. Applying the same argument when $j=m$,

$$
g_{i} \in g_{i+1} C \cup \cdots \cup g_{i+m} C,
$$

so every $g_{i}$ belongs to the $C$-neighborhood of some element of $\left\{g_{i+1}, \ldots, g_{i+m}\right\}$.

For $u$ and $v$ positive integers $u<v$, let $I[u, v]$ denote the set of integers $u \leqslant x \leqslant v$. Consider the transitive closure of the relation $\sim_{C}$ on $I[u, v]$ given by $g_{i} \in g_{j} C$. Thus, $g_{i} g_{j}^{-1} \in C^{v-u}$ whenever 


\section{LARSEn And V. A. Lunts}

$i$ and $j$ belong to the same equivalence class. By (3) and (4), every $m$-interval in $I[u, v]$ contains a representative of each $\sim_{C}$ equivalence class, so there are at most $m$ such classes. If $f(t)$ had dispersion $\geqslant m+1$, we could choose $N$ as in Definition 2.8, set $K=C^{N}$, and choose an $(m+1)$ element set $S \subset I[u, u+N]$ in contradiction to our upper bound for equivalence classes in $I[u, u+N]$. It follows that $f(t)$ has dispersion $r \leqslant m$.

In particular, $f(t)$ has dispersion $\geqslant r$, and we fix $N \geqslant m$ as in Definition 2.8. As the dispersion of $f$ is $\leqslant r$, there exists a finite set $K$ (which we may assume contains $C$ and is closed under inverses) such that for every $(r+1)$-element subset $S$ of an $N$-interval

$$
\exists s, t \in S, s \neq t, g_{s} \in g_{t} K .
$$

On the other hand, for every integer $M \geqslant N$, there exist integers $u$ and $i_{1}, \ldots, i_{r}$ such that

$$
u=i_{1}<i_{2}<\cdots<i_{r} \leqslant u+N
$$

and no $g_{i_{j}}$ lies within the $K^{M}$-neighborhood of another $g_{i_{k}}$. We consider the equivalence relation $\sim_{K}$ on $I[u, u+M]$. The integers $i_{1}, \ldots, i_{r}$ belong to distinct equivalence classes in the interval $I[u, u+M]$. As each $m$-interval in $I[u, u+M]$ contains a representative of each $\sim_{C}$ equivalence class, every $N$-interval in $I[u, u+M]$ contains at least one representative of each $\sim_{K}$ equivalence class. By (5), every $g_{j}$ with $j \in[u, u+N]$ lies within the $K$-neighborhood of some $g_{i_{k}}$; it follows that every $g_{j}$ with $j \in[u, u+M]$ lies in the $\sim_{K}$ equivalence class of some $g_{i_{k}}$. The $\sim_{K}$ equivalence classes of the $g_{i_{k}}$ therefore partition $I[u, u+M]$.

For $j \in I[u, u+M]$ and $1 \leqslant k \leqslant r$, define

$$
g_{j, k}= \begin{cases}g_{j} & \text { if } j \text { is } \sim_{K} \text { equivalent to } i_{k}, \\ 0 & \text { otherwise. }\end{cases}
$$

Thus $g_{j}=\sum_{k=1}^{r} g_{j, k}$. For $j \in I[u, u+M-m]$, therefore,

$$
\sum_{k=1}^{r}\left(b_{0} g_{j+m, k}+b_{1} g_{j+m-1, k}+\cdots+b_{m} g_{j, k}\right)=0 .
$$

There can be no common monomial appearing in the sums $b_{0} g_{j+m, k}+\cdots+b_{m} g_{j, k}$ for two different values of $k$. It follows that

$$
b_{0} g_{j+m, k}+b_{1} g_{j+m-1, k}+\cdots+b_{m} g_{j, k}=0,
$$

and this implies that, for fixed $k$ and variable $j \in I[u, u+M-m]$, the $g_{j, k}$ satisfy the same $m$ th-order linear recurrence relation as the original sequence of coefficients $g_{j}$.

For each $k$, every non-zero $g_{j, k}, j \in I[u, u+M]$, lies within the $K^{M}$-neighborhood of $g_{i_{k}}$. Suppose $j_{1}, j_{2} \in I[u, u+M]$ are equivalent to one another and lie in the same $N$-interval $J \subset$ $I[u, u+M]$. Suppose they are both equivalent to $i_{k}$. For each $l \neq k$, we choose $i_{l}^{\prime} \in J$ equivalent to $i_{l}$. Thus $g_{i_{l}^{\prime}}$ is within the $K^{M}$-neighborhood of $g_{i_{l}}$. Applying (5) to $\left\{j_{1}, j_{2}, i_{1}^{\prime}, \ldots, i_{k-1}^{\prime}, i_{k+1}^{\prime}, \ldots, i_{r}^{\prime}\right\}$, we conclude that $j_{1} \in j_{2} K$. Since the $g_{j, k}$ satisfy an $m$ th-order linear recurrence relation (6), any two consecutive non-zero terms in the sequence must lie in a common $m$-interval and therefore in a common $N$-interval. Thus, any two consecutive non-zero terms in the sequence

$$
g_{u, k}, g_{u+1, k}, \ldots, g_{u+M, k}
$$

lie in one another's $K$-neighborhoods. It follows that, up to scalar multiplication by an element of $G$, there are only a bounded number of possibilities for an $m$-tuple consisting of $m$ consecutive terms of (7), where the bound does not depend on $M$. If $M$ is taken sufficiently large, we can find integers $a$ and $p$ and elements $c_{k} \in G$ such that $u \leqslant a<a+p \leqslant u+M-m$, and for each $k$, $1 \leqslant k \leqslant r$

$$
c_{k}\left(g_{a, k}, g_{a+1, k}, \ldots, g_{a+m-1, k}\right)=\left(g_{a+p, k}, g_{a+p+1, k}, \ldots, g_{a+p+m-1, k}\right) .
$$




\section{RATIONALITY CRITERIA FOR MOTIVIC ZETA FUNCTIONS}

The linear recurrence (6) and induction imply

$$
g_{i+p, k}=c_{k} g_{i, k}
$$

for all $i \in I[a, u+M-p]$. Thus, for $i$ in this interval, the unique value of $k$ for which $g_{i, k} \neq 0$ depends only on $i(\bmod p)$. In particular, for each residue class $(\bmod p)$ there is exactly one value of $k$ for which $g_{j, k} \neq 0$ for $j \in I[a, u+M]$ belonging to this residue class. For $a \leqslant j<a+p$ and $s$ non-negative, we define

$$
g_{j+s p, k}^{\prime}=c_{k}^{s} g_{j, k}
$$

Thus $g_{j, k}^{\prime}$ coincides with $g_{j, k}$ for $j \in I[a, u+M]$. Since $g_{j, k}^{\prime}$ satisfies the same $m$ th-order recurrence as $g_{j, k}$ for all $j \in I[a, a+p-1]$, it does so for all $j$. Defining

$$
g_{j}^{\prime}=\sum_{k=1}^{r} g_{j, k}^{\prime},
$$

the sequence $g_{j}^{\prime}$ again satisfies the same $m$ th-order recurrence as $g_{j}$. Since $g_{j}=g_{j}^{\prime}$ for all $j \in$ $I[a, a+m-1]$, we have $g_{j}=g_{j}^{\prime}$ for all $j \geqslant a$, and setting $i_{0}=a$ and $h_{i}=c_{k}$ for the equivalence class $k$ corresponding to the residue class of $i(\bmod p)$, the theorem follows.

\section{Rationality theorems}

In this section we describe several classes of varieties that have rational zeta functions, notably rational and ruled surfaces. These are rather special cases, and indeed we suspect, that except in dimension 1, rationality of motivic zeta functions is the exception rather than the rule. Some evidence for this belief is given in the discussion of irrationality theorems in $\S 7$.

Lemma 3.1. If $X$ is a variety and $Y$ a closed subvariety with complement $U$, then if any two of $\zeta_{X}(t), \zeta_{Y}(t)$, and $\zeta_{U}(t)$ are globally (respectively pointwise) rational, then the third is so as well.

Proof. Stratifying $\operatorname{Sym}^{n} X$ according to how many points land in $Y$, we obtain

$$
\left[\operatorname{Sym}^{n} X\right]=\sum_{i+j=n}\left[\operatorname{Sym}^{i} Y \times \operatorname{Sym}^{j} U\right]=\sum_{i+j=n}\left[\operatorname{Sym}^{i} Y\right]\left[\operatorname{Sym}^{j} U\right] .
$$

It follows that $\zeta_{X}(t)=\zeta_{Y}(t) \zeta_{U}(t)$.

By Lemma 2.5, not only are the two specified zeta functions globally (respectively pointwise) rational, the same is true of their reciprocals. The product of rational zeta functions is again rational, and the lemma follows.

In particular, the disjoint union of varieties with globally rational zeta functions again has a globally rational zeta function. This shows in particular that zero-dimensional varieties have globally rational zeta functions; explicitly, the zeta function of a variety with $n$ points is $(1-t)^{-n}$. Also, a stratified variety has such a zeta function as long as all of its strata do.

Lemma 3.2. If $X$ is any variety, and $E \rightarrow X$ is a fiber bundle, locally trivial in the Zariski topology, with fiber $F$, then $[E]=[X][F]$. In particular, if $E \rightarrow X$ is a vector bundle of rank $r$, then

$$
[E]=[X]\left[\mathbb{A}^{r}\right] .
$$

Proof. Note first of all that vector bundles are Zariski locally trivial, so the second claim is indeed a special case of the first. As $E \rightarrow X$ is locally trivial, there exists a dense open subset $U \subset E$ over which $E$ restricts to a trivial bundle. If $Y$ denotes the complement of $U$ and $E_{U}$ (respectively $E_{Y}$ ) the pull-back of $E$ to $U$ (respectively $Y$ ) respectively, it suffices to prove the lemma for $E_{Y} \rightarrow Y$ and $E_{U} \rightarrow U$. As $E_{U}=U \times F$, the lemma follows by Noetherian induction. 


\section{LARSEN AND V. A. Lunts}

The following proposition is due to B. Totaro (see [Göt01]).

Proposition 3.3. If $X$ is any variety, $E \rightarrow X$ is a vector bundle of rank $r$, and $n$ is any positive integer, then $\left[\operatorname{Sym}^{n} E\right]=\left[\operatorname{Sym}^{n} X\right]\left[\mathbb{A}^{1}\right]^{r n}$. Equivalently, $\zeta_{E}(t)=\zeta_{X}\left(\left[\mathbb{A}^{r}\right] t\right)$.

Corollary 3.4. If $X$ is a variety such that $\zeta_{X}(t)$ is globally (respectively pointwise) rational, and $E \rightarrow X$ is a vector bundle, then $\zeta_{E}(t)$ is globally (respectively pointwise) rational.

COROllary 3.5. For all non-negative integers $n, \zeta_{\mathbb{A}^{n}}(t)$ is rational.

Corollary 3.6. If $X$ is a variety and $P \rightarrow X$ is a projective space bundle of rank $r$ that is locally trivial in the Zariski topology, then

$$
\zeta_{P}(t)=\zeta_{X}(t) \zeta_{X}\left(\left[\mathbb{A}^{1}\right] t\right) \cdots \zeta_{X}\left(\left[\mathbb{A}^{r}\right] t\right) .
$$

In particular, $\zeta_{\mathbb{P} r}(t)$ is rational for all $r$.

Proof. By Noetherian induction, it suffices to consider the case $P=X \times \mathbb{P}^{r}$. The corollary then follows immediately from the stratification of $\mathbb{P}^{r}$ with strata $\mathbb{A}^{i}, 0 \leqslant i \leqslant r$.

We come now to the main positive result in the subject.

Theorem 3.7 (Kapranov). If $X$ is any one-dimensional variety, then there exist positive integers $m$ and $n$ such that $(1-t)^{m}\left(1-\left[\mathbb{A}^{1}\right] t\right)^{n} \zeta_{X}(t)$ is a polynomial. In particular, $\zeta_{X}(t)$ is globally rational.

Proof. Let $X$ be a one-dimensional variety. The singular locus $Y$ is either empty or zero-dimensional. In the latter case, the question reduces to the case of $X \backslash Y$, so without loss of generality we may assume $X$ is non-singular. If $X$ has more than one component, it suffices to prove the theorem for each one. Without loss of generality, we may assume $X$ is connected and therefore irreducible. Let $\bar{X}$ denote the unique projective non-singular curve containing $X$ as an open subvariety. As $\bar{X} \backslash X$ is empty or zero-dimensional, without loss of generality we may assume that $X$ is projective and non-singular.

Let $g$ be the genus of $X$. Let $x_{0}$ denote a base point of $X$ and $J$ denote the Jacobian variety Jac $X$. For non-negative $n \geqslant 2 g-1$, the morphism $X^{n} \rightarrow J$ mapping

$$
\left(x_{1}, \ldots, x_{n}\right) \mapsto-n x_{0}+\sum_{i=1}^{n} x_{i}
$$

factors through $\operatorname{Sym}^{n} X$, realizing it as a projective space bundle of rank $n-g$ over $J$. This bundle is the projectivization of a natural rank- $(n+1-g)$ vector bundle on $J$, so by Lemma 3.2,

$$
\left[\operatorname{Sym}^{n} X\right]=[J]\left(1+\left[\mathbb{A}^{1}\right]+\cdots+\left[\mathbb{A}^{n-g}\right]\right) .
$$

This implies that

$$
\zeta_{X}(t)(1-t)\left(1-\left[\mathbb{A}^{1}\right] t\right)
$$

is a polynomial of degree $\leqslant 2 g$.

COROLlary 3.8. If $X$ is an algebraic surface, the global (respectively pointwise) rationality of $\zeta_{X}(t)$ depends only on the birational equivalence class of $X$.

Proof. If $X_{1}$ and $X_{2}$ are two such surfaces and $U$ is a surface that is isomorphic to dense open subvarieties of each, then setting $Y_{i}=X_{i} \backslash U$,

$$
\zeta_{X_{i}}(t)=\zeta_{U}(t) \zeta_{Y_{i}}(t)
$$

By Theorem 3.7, $\zeta_{Y_{i}}(t)$ is globally (therefore also pointwise) rational, so by Lemma 3.1, each $\zeta_{X_{i}}(t)$ is rational if and only if $\zeta_{U}(t)$ is so. 


\section{RATIONALITY CRITERIA FOR MOTIVIC ZETA FUNCTIONS}

Theorem 3.9. If $X$ is a surface with Kodaira dimension $-\infty$, then $\zeta_{X}(t)$ is globally rational.

Proof. There are two cases: rational surfaces and birationally ruled surfaces. In each case, we may choose any variety in the given birational equivalence class. For rational surfaces, we use $\mathbb{A}^{2}$, which has a globally rational zeta function by Corollary 3.5. A ruled surface is a projective line bundle over a curve. By the Tsen-Lang theorem, such a $\mathbb{P}^{1}$-bundle is Zariski locally trivial, and by Theorem 3.7 and Corollary 3.6, the motivic zeta function of a ruled surface is globally rational.

\section{Lambda rings}

In this section, we develop some basic definitions and facts connected with the notion of $\lambda$-ring. A good reference for this material is the first section of [AT69].

Definition 4.1. A $\lambda$-structure on a commutative $\operatorname{ring} A$ is an infinite sequence $\lambda^{0}, \lambda^{1}, \lambda^{2}, \ldots$, of maps $A \rightarrow A$ such that

$$
\begin{gathered}
\lambda^{0}(x)=1, \\
\lambda^{1}(x)=x, \\
\lambda^{n}(x+y)=\sum_{i+j=n} \lambda^{i}(x) \lambda^{j}(y) .
\end{gathered}
$$

A $\lambda$-ring is a commutative ring endowed with a $\lambda$-structure. We call a ring homomorphism between $\lambda$-rings that commutes with $\lambda$-operations a $\lambda$-homomorphism.

The prototype of a $\lambda$-ring is the Grothendieck ring of (virtual) finite-dimensional vector spaces over a field, where $\lambda^{i}$ gives the $i$ th exterior power. Of course, explicitly the ring here is $\mathbb{Z}$, and $\lambda^{i}(n)=\left(\begin{array}{c}n \\ i\end{array}\right)$. More generally, one can look at Grothendieck rings of finite-dimensional representations of a group, finitely generated projective modules of a ring or finite-rank vector bundles over a variety or a topological space.

Definition 4.2. Given a $\lambda$-structure on a ring $A$, we will call the additive-to-multiplicative group homomorphism $\lambda_{t}: A \rightarrow 1+t A[[t]]$ given by

$$
\lambda_{t}(a)=\sum_{n=0}^{\infty} \lambda^{n}(a) t^{n} .
$$

the universal $\lambda$-homomorphism.

Every $\lambda$-structure defines a homomorphism $\lambda_{t}$; conversely, every homomorphism $A \rightarrow 1+t A[[t]]$ that is congruent to $a \mapsto 1+a t\left(\bmod t^{2}\right)$ defines a $\lambda$-structure.

Definition 4.3. If $\lambda_{t}$ is the universal $\lambda$-homomorphism associated to a $\lambda$-structure, then the $\lambda$-structure $\sigma^{0}, \sigma^{1}, \ldots$ associated to the homomorphism $\sigma_{t}(a):=\lambda_{-t}(-a)=\lambda_{-t}(a)^{-1}$ is called the opposite structure to $\lambda^{0}, \lambda^{1}, \ldots$

For example, if $A$ is the Grothendieck ring of vector spaces (or representations, projective modules, vector bundles, etc.) and the original $\lambda$-structure gives exterior powers, then the opposite $\lambda$-structure gives symmetric powers. Passage to the opposite is involutive; this is obvious on the level of universal $\lambda$-homomorphisms. The identities

$$
\lambda^{n}(x-y)=\sum_{i+j=n}(-1)^{j} \lambda^{i}(x) \sigma^{j}(y), \quad \sigma^{n}(x-y)=\sum_{i+j=n}(-1)^{j} \sigma^{i}(x) \lambda^{j}(y)
$$

are immediate from the definitions and indicate what is meant explicitly by an exterior or symmetric power of a virtual vector space (or representation, projective module, vector bundle, etc.). 


\section{LARSEN AND V. A. Lunts}

Lemma 4.4. Let $A$ and $B$ denote $\lambda$-rings and $\phi: A \rightarrow B$ a ring homomorphism. Then the set of elements of $A$ for which $\phi$ commutes with all $\lambda$-operations is an additive subgroup.

Proof. As $\phi$ is a ring homomorphism, it defines a multiplicative group homomorphism

$$
f: 1+t A[[t]] \rightarrow 1+t B[[t]] .
$$

The subset of $A$ on which the diagram of commutative groups and group homomorphisms

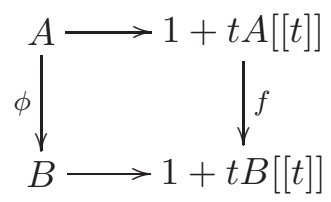

commutes is therefore a subgroup of $A$.

Definition 4.5. An element $a \in A$ is finite-dimensional if $\lambda_{t}(a)$ is a polynomial, and the dimension of $a$ is the degree of this polynomial. A difference of finite-dimensional elements is virtually finite. A $\lambda$-ring is finite-dimensional if all of its elements are virtually finite.

Note that $\lambda_{t}$ of a virtually finite element is a rational function in $t$.

The Grothendieck ring of finite-dimensional vector spaces (or representations, projective modules, vector bundles, etc.) is finite-dimensional. An element $x$ is finite with respect to a $\lambda$-structure on $A$ if and only if $-x$ is finite with respect to the opposite $\lambda$-structure, so the group of virtually finite elements is invariant under passage to opposites. Note also that, in any $\lambda$-ring $A, \lambda_{t}$ of a virtually finite element must be a ratio of polynomials with constant term 1 and therefore a globally rational element of $A[[t]]$.

Next, we will consider some slightly more involved examples of $\lambda$-rings.

Example 4.6. Consider the polynomial $\operatorname{ring} \mathbb{Z}[s]$. If we identify $\mathbb{Z}$ with the ring of virtual finitedimensional vector spaces, then $\mathbb{Z}[s]$ is identified with the ring of isomorphism classes of $\mathbb{N}$-graded virtual finite-dimensional vector spaces. Using this identification, define the $\lambda$-structure on $\mathbb{Z}[s]$ as follows:

$$
\lambda^{i}\left(V s^{p}\right)= \begin{cases}\operatorname{Sym}^{i} V s^{i p}, & \text { if } p \text { is even, } \\ \Lambda^{i} V s^{i p}, & \text { if } p \text { is odd. }\end{cases}
$$

Note that $\lambda^{i}$ corresponds to the usual $i$ th symmetric power of a virtual super vector space.

Example 4.7. Let $A$ be a $\lambda$-ring and $M \subset A$ a multiplicative submonoid closed under the $\lambda$-operations. Consider the corresponding monoid ring $\mathbb{Z}[M]$. (Note that it is not a subring of $A$.) Then $\mathbb{Z}[M]$ has a natural $\lambda$-structure given by

$$
\lambda^{i}([m])=\left[\lambda^{i} m\right] .
$$

Example 4.8. Let us combine the two previous examples. Let $M \subset \mathbb{Z}[s]$ be the multiplicative monoid, which consists of polynomials with constant term 1 . Then $\mathbb{Z}[M]$ is a $\lambda$-ring. This example will be important to us since our motivic measures will take their values in $\mathbb{Z}[M]$ or, more precisely, its fraction field. The fraction field is well defined because of the following lemma.

Lemma 4.9. The monoid $M$ is a free commutative monoid. The ring $\mathbb{Z}[M]$ is a polynomial ring, hence an integral domain.

Proof. The ring $\mathbb{Z}[s]$ is factorial and any element of $M$ is a unique product of elements of $M$, which are prime in $\mathbb{Z}[s]$ (the only unit in $M$ is 1 ). Thus $M$ is isomorphic to the monoid $\oplus \mathbb{N}$, where the summation is over all prime elements of $\mathbb{Z}[s]$ which are in $M$. Hence, $\mathbb{Z}[M]$ is a polynomial ring, so it is an integral domain. 


\section{RATIONALITY CRITERIA FOR MOTIVIC ZETA FUNCTIONS}

Example 4.10. By Equation (8), the Grothendieck ring of varieties $K_{0}\left[\mathcal{V}_{K}\right]$ has a natural $\lambda$-ring structure for any field $K$ :

$$
\lambda_{t}([X])=\zeta_{X}(t)
$$

Thus $\lambda^{i}[X]:=\left[\operatorname{Sym}^{i} X\right]$.

For any commutative ring $A$ there is a natural $\lambda$-ring structure on the set $1+t A[[t]]$. The operation of addition in this ring is given by the multiplication of power series. Multiplication $(\cdot)$ and the $\lambda$-operations $\left(\Lambda^{i}\right)$ are given by universal polynomials which are uniquely characterized by the identities

$$
\left\{\prod_{i=1}^{m}\left(1+a_{i} t\right)\right\} \cdot\left\{\prod_{j=1}^{n}\left(1+b_{j} t\right)\right\}=\prod_{i=1}^{m} \prod_{j=1}^{n}\left(1+a_{i} b_{j} t\right)
$$

and

$$
\Lambda^{k} \prod_{i=1}^{n}\left(1+a_{i} t\right)=\prod_{\substack{S \subset\{1, \ldots, n\},|S|=k}}\left(1+t \prod_{j \in S} a_{j}\right) .
$$

Equation (9) implies that the polynomial expressing the $t^{p}$ coefficient of $\sum_{i} x_{i} t^{i} \cdot \sum_{j} y_{j} t^{j}$ in terms of $x_{i}$ and $y_{j}$ lies in the ideal

$$
\left(x_{m+1}, x_{m+2}, \ldots, y_{n+1}, y_{n+2}, \ldots\right)
$$

whenever $p>m n$. This implies that the product of two polynomials in $1+t A[[t]]$ is again a polynomial, regardless of whether the polynomials split into linear factors. As $\cdot$ distributes over the usual multiplication of power series, $f(t) \cdot g(t)$ is a ratio of polynomials in $1+t A[[t]]$ if $f(t)$ and $g(t)$ are.

Definition 4.11. We say that $A$ is a special $\lambda$-ring if $\lambda_{t}$ is a homomorphism not merely of groups but of $\lambda$-rings.

For example, the Grothendieck group of vector spaces (or representations, projective modules, or vector bundles) is a special $\lambda$-ring. Moreover, $1+t A[[t]]$ is always special, regardless of whether or not $A$ is so. On the other hand, Examples 4.6, 4.8, and 4.10 are not special, and neither is Example 4.7 in general, even if $A$ happens to be so. Special $\lambda$-rings are characterized by identities of the form

$$
\lambda^{n}(x y)=P_{n}\left(x, \lambda^{2} x, \ldots, \lambda^{n} x, y, \ldots, \lambda^{n} y\right)
$$

and

$$
\lambda^{m} \lambda^{n} x=P_{m, n}\left(x, \lambda^{2} x, \ldots, \lambda^{m n} x\right)
$$

for certain universal polynomials $P_{n}, P_{m, n}$. Equation (10) guarantees that $\lambda_{t}$ is a ring homomorphism and (11) guarantees that it respects $\lambda$-structures.

Note that the set of virtually finite elements in a special $\lambda$-ring forms a $\lambda$-subring.

Lemma 4.12. For any $\lambda$-ring $A$ there exists a universal pair consisting of a special $\lambda$-ring $B$ and a $\lambda$-homomorphism $A \rightarrow B$ such that every $\lambda$-homomorphism from $A$ to a special $\lambda$-ring $C$ factors through $B$.

Proof. If $C$ is a special $\lambda$-ring and $\Phi: A \rightarrow C$ is a $\lambda$-homomorphism, then for all $x, y \in A$,

$$
\begin{aligned}
\Phi\left(\lambda^{n}(x y)\right)=\lambda^{n} \Phi(x y) & =\lambda^{n}(\Phi(x) \Phi(y)) \\
& =P_{n}\left(\Phi(x), \ldots, \lambda^{n} \Phi(x), \Phi(y), \ldots, \lambda^{n} \Phi(y)\right) \\
& =P_{n}\left(\Phi(x), \ldots, \Phi\left(\lambda^{n} x\right), \Phi(y), \ldots, \Phi\left(\lambda^{n} y\right)\right) \\
& =\Phi\left(P_{n}\left(x, \ldots, \lambda^{n} x, y, \ldots, \lambda^{n} y\right)\right),
\end{aligned}
$$


So

$$
\lambda^{n}(x y)-P_{n}\left(x, \ldots, \lambda^{n} x, y, \ldots, \lambda^{n} y\right) \in \operatorname{ker} \Phi .
$$

Similarly,

$$
\lambda^{m} \lambda^{n} x-P_{m, n}\left(x, \ldots, \lambda^{m n} x\right) \in \operatorname{ker} \Phi .
$$

Let $I$ denote the $\lambda$-ideal in $A$ generated by elements of type (12) and (13). Thus $I \subset \operatorname{ker} \Phi$. Conversely, if $B=A / I$, then (12) and (13) imply (10) and (11), respectively. Thus, the quotient map $A \rightarrow B$ is universal.

We call the ring $B$ the specialization of $A$.

The opposite of a special $\lambda$-ring need not be special. For example, in $\mathbb{Z}$, which is special with respect to $\left\{\lambda^{n}\right\}$,

$$
\sigma^{n}(r)=\left(\begin{array}{c}
r+n-1 \\
n
\end{array}\right)
$$

so the $\left\{\sigma^{n}\right\}$ counterpart of the identity (10) for $n=2$, namely

$$
\sigma^{2}(x y)=y^{2} \sigma^{2} x+x^{2} \sigma^{2} y-2\left(\sigma^{2} x\right)\left(\sigma^{2} y\right)
$$

(see [BGI77, V 2.3]) does not hold.

Finally, we mention the Adams operations $\Psi^{n}: A \rightarrow A$ defined on a special $\lambda$-ring. They are given in terms of $\lambda$-operations by the identity

$$
\sum_{n=1}^{\infty} \Psi^{n}(a)(-t)^{n}=t \lambda_{t}(a)^{-1} \frac{d}{d t} \lambda_{t}(a)
$$

(see [AT69, ch. I, $\S 5]$, but note the sign error therein). The Adams operations commute with both products and $\lambda$-operations and therefore define $\lambda$-ring endomorphisms [AT69, I, Proposition 5.1].

\section{Special $\lambda$-ring $\bar{K}(X)$}

Let $X$ be a variety. It is well known that the usual $K$-theory (of algebraic vector bundles) associates to $X$ a special $\lambda$-ring $K(X)$. We will need to consider a different Grothendieck group $\bar{K}(X)$ of vector bundles, so that the functor of global sections descends to a group homomorphism

$$
H_{X}^{0}: \bar{K}(X) \rightarrow K[\text { Vect] }
$$

So let us take $\bar{K}(X)$ to be the abelian group generated by isomorphism classes of algebraic vector bundles on $X$ with relations

$$
[P]=[M]+[N],
$$

whenever the vector bundles $M \oplus N$ and $P$ are isomorphic. Note that we do not impose relations coming from general short exact sequences as in the usual $K$-theory. The $\otimes$ operation makes $\bar{K}(X)$ a ring and the $\lambda$-operations are defined in the usual way using the exterior powers:

$$
\lambda^{i}[P]=\left[\Lambda^{i} P\right] .
$$

The traditional proof that $K(X)$ is a special $\lambda$-ring uses the splitting principle. The usual method of splitting an algebraic vector bundle produces only a short exact sequence and is not therefore applicable to our group $\bar{K}(X)$. Nevertheless, we have the following theorem.

TheOREM 5.1. The $\lambda$-ring $\bar{K}(X)$ is special.

Proof. We will prove the identities (10) and (11) in $\bar{K}(X)$ by showing that for any $x, y \in \bar{K}(X)$ there exists a $\lambda$-homomorphism from a special $\lambda$-ring to $\bar{K}(X)$ such that $x, y$ are contained in the image. For this we need a free special $\lambda$-ring on two generators. 


\section{RATIONALITY CRITERIA FOR MOTIVIC ZETA FUNCTIONS}

First recall the free special $\lambda$-ring on one generator. It has at least three well-known descriptions. First, it can be described as the ring of symmetric functions in an infinite number of variables [AT69, ch. I, $\S 2]$, where $\lambda^{i} x$ corresponds to the $i$ th elementary symmetric function. By the fundamental theorem on symmetric polynomials, this is isomorphic to $\mathbb{Z}\left[\lambda^{1} x, \lambda^{2} x, \lambda^{3} x, \ldots\right]$. Secondly, it can be described as the direct sum of the representation rings of all symmetric groups $S_{n}$. Thirdly, it can be described as the ring of polynomial functors on the category of vector spaces over an arbitrary field in characteristic 0 (or more precisely, the ring associated to the semiring of polynomial functors). The equivalence of the first and second definitions is classical (see, e.g., [Knu73, p. 135]). The equivalence of the second and third definitions is given in [Mac95, ch. I, Appendix A, § 5]. Explicitly a rational representation $V$ of $S_{n}$ gives rise to the functor $M \mapsto M^{\otimes n} \otimes_{\mathbb{Q}\left[S_{n}\right]} V$, which we abbreviate to $M \mapsto M^{\otimes n} \otimes_{S_{n}} V$. On the level of functors, the meaning of product and $\lambda^{i}$ is obvious, and this dictates the definition on the level of representations of symmetric groups. These definitions are given explicitly in [Mac95, ch. I, Appendix A, § 6], and we now recall them.

Let $R_{n}$ be the representation ring of the symmetric group $S_{n}$, with the convention that $S_{0}=\{e\}$. That is, $R_{n}$ is a free $\mathbb{Z}$-module with basis consisting of isomorphism classes of rational irreducible representations of $S_{n}$ (or equivalently, isomorphism classes of complex irreducible representations). Put

$$
R:=\bigoplus_{n \geqslant 0} R_{n}
$$

Thus $R$ has a $\mathbb{Z}$-basis consisting of pairs $(n, \omega)$, where $n$ is a natural number and $\omega$ is a rational irreducible representation of $S_{n}$. The ring structure is given explicitly by the formula (see [Knu73, p. 127] or [Mac95, ch. I, Appendix A, (6.1)])

$$
\left(n_{1}, \omega_{1}\right)\left(n_{2}, \omega_{2}\right)=\left(n_{1}+n_{2}, \operatorname{Ind}_{S_{n_{1}} \times S_{n_{2}}}^{S_{n_{1}+n_{2}}} \omega_{1} \otimes \omega_{2}\right) .
$$

The trivial $S_{0}=\{e\}$-module is the unit in the $\operatorname{ring} R$. The $\lambda$-operations are defined as follows [Mac95, ch. I, Appendix A, (6.2)]. Fix a basis element $(n, \omega)$ and a positive integer $r$. Consider the obvious action of the wreath product $S_{n}^{r} \rtimes S_{r}$ on $\omega^{\otimes r}$ and let $\operatorname{Sign}\left(S_{r}\right)$ be the sign representation of $S_{r}$. Then $\omega^{\otimes r} \otimes \operatorname{Sign}\left(S_{r}\right)$ is a left $S_{n}^{r} \rtimes S_{r}$-module, and

$$
\lambda^{r}(n, \omega)=\left(r n, \operatorname{Ind}_{S_{n}^{r} \rtimes S_{r}}^{S_{r n}} \omega^{\otimes r} \otimes \operatorname{Sign}\left(S_{r}\right)\right) .
$$

As a special $\lambda$-ring, $R$ is generated by the element $(1, \mathbb{Q})$. The ring $R$ is free in the obvious sense: given a special $\lambda$-ring $Q$ and an element $x \in Q$, there exists a unique $\lambda$-homomorphism $f: R \rightarrow Q$ such that $f((1, \mathbb{Q}))=x$.

Consider the special $\lambda$-ring $R^{2}:=R \otimes_{\mathbb{Z}} R$. It is the free special $\lambda$-ring on two generators in the obvious sense. Naturally $R^{2}$ has a $\mathbb{Z}$-basis consisting of elements $\left(\left(n_{1}, n_{2}\right), \omega_{1} \otimes \omega_{2}\right)$, where $\omega_{i}$ is an irreducible representation of $S_{n_{i}}, i=1,2$; we regard $\omega_{1} \otimes \omega_{2}$ as an $S_{n_{1}} \times S_{n_{2}}$-representation in the usual way. The $\lambda$-operations are similar to those in $R$ :

$$
\lambda^{r}\left(\left(n_{1}, n_{2}\right), \omega_{1} \otimes \omega_{2}\right)=\left(\left(r n_{1}, r n_{2}\right), \operatorname{Ind}_{\left(S_{n_{1}}^{r} \times S_{n_{2}}^{r}\right) \rtimes S_{r}}^{S_{r n_{1}} \times S_{n_{2}}} \omega_{1}^{\otimes r} \otimes \omega_{2}^{\otimes r} \otimes \operatorname{Sign}\left(S_{r}\right)\right) .
$$

For any vector bundle $M$ on $X$, we define

$$
\lambda_{t}([M])=\sum_{i=0}^{\infty}\left[\Lambda^{i} M\right] t^{i} .
$$

It is easy to check that this extends uniquely to a well-defined $\lambda$-structure on $\bar{K}(X)$.

For fixed vector bundles $M$ and $N$ we define $\Theta: R^{2} \longrightarrow \bar{K}(X)$ as follows:

$$
\Theta\left(\left(n_{1}, n_{2}\right), \omega_{1} \otimes \omega_{2}\right)=\left[\left(M^{\otimes n_{1}} \otimes N^{\otimes n_{2}}\right) \otimes S_{n_{1}} \times S_{n_{2}}\left(\omega_{1} \otimes \omega_{2}\right)\right] .
$$




\section{LARSEn AND V. A. Lunts}

It is clear that $\Theta$ defines a ring homomorphism. We claim that $\Theta$ respects $\lambda$-structures. If true, this means that the $\lambda$-ring identities (10) and (11) hold for all classes $x$ and $y$ of effective vector bundles on $x$. In other words, $\lambda_{t}: \bar{K}(X) \rightarrow \bar{K}(X)[[t]]$ satisfies the identities

$$
\lambda_{t}(x y)=\lambda_{t}(x) \cdot \lambda_{t}(y), \quad \lambda_{t}\left(\lambda^{r} x\right)=\Lambda^{r} \lambda_{t}(x)
$$

when $x$ and $y$ are effective classes. As $\lambda_{t}(x y)-\lambda_{t}(x) \cdot \lambda_{t}(y)$ is bi-additive and zero on a set spanning $\bar{K}(X)$, it is identically 0 , and $\lambda_{t}$ is a ring homomorphism. Applying Lemma 4.4 to $\lambda_{t}$ itself, we conclude that the set of elements on which $\lambda_{t}$ commutes with $\lambda$-operations is all of $\bar{K}(X)$. To prove the theorem, therefore, it suffices to prove our claim that $\Theta$ respects $\lambda$-structures; that is, we need to compare

$$
\begin{aligned}
& \Theta\left(\lambda^{r}\left(\left(n_{1}, n_{2}\right), \omega_{1} \otimes \omega_{2}\right)\right) \\
& \quad=\left(M^{\otimes r n_{1}} \otimes N^{\otimes r n_{2}}\right) \otimes_{S_{r n_{1}} \times S_{r n_{2}}}\left(\mathbb{Q}\left[S_{r n_{1}} \times S_{r n_{2}}\right] \otimes_{\left(S_{n_{1}}^{r} \times S_{n_{2}}^{r}\right) \rtimes S_{r}}\left(\omega_{1}^{\otimes r} \otimes \omega_{2}^{\otimes r} \otimes \operatorname{Sign}\left(S_{r}\right)\right)\right) \\
& \quad=\left(M^{\otimes r n_{1}} \otimes N^{\otimes r n_{2}}\right) \otimes_{\left(S_{n_{1}}^{r} \times S_{n_{2}}^{r}\right) \rtimes S_{r}}\left(\omega_{1}^{\otimes r} \otimes \omega_{2}^{\otimes r} \otimes \operatorname{Sign}\left(S_{r}\right)\right)
\end{aligned}
$$

and

$$
\Lambda^{r}\left(\Theta\left(\left(n_{1}, n_{2}\right), \omega_{1} \otimes \omega_{2}\right)\right)=\Lambda^{r}\left(\left(M^{\otimes n_{1}} \otimes N^{\otimes n_{2}}\right) \otimes_{S_{n_{1}} \times S_{n_{2}}}\left(\omega_{1} \otimes \omega_{2}\right)\right) .
$$

The following lemma implies that the two are isomorphic.

Lemma 5.2. Let $G$ be a group, and let $P$ and $Q$ be right and left $G$-modules respectively. Consider the space $\left(P \otimes_{G} Q\right)^{\otimes r}=P^{\otimes r} \otimes_{G^{r}} Q^{\otimes r}$ as a right $S_{r}$-module. There is a canonical isomorphism of right $S_{r}$-modules

$$
\alpha: P^{\otimes r} \otimes_{G^{r}} Q^{\otimes r} \stackrel{\sim}{\longrightarrow} P^{\otimes r} \otimes_{G^{r} \rtimes S_{r}}\left(Q^{\otimes r} \otimes \mathbb{Q}\left[S_{r}\right]\right) .
$$

In particular there is a canonical isomorphism of vector spaces

$$
\Lambda^{r}\left(\left(P \otimes_{G} Q\right)^{\otimes r}\right) \stackrel{\sim}{\longrightarrow} P^{\otimes r} \otimes_{G^{r} \rtimes S_{r}}\left(Q^{\otimes r} \otimes \operatorname{Sign}\left(S_{r}\right)\right) .
$$

Proof. Indeed,

$$
\alpha: x \otimes y \mapsto x \otimes y \otimes 1,
$$

and

$$
p_{1} \otimes \cdots \otimes p_{r} \otimes q_{1} \otimes \cdots \otimes q_{r} \otimes \tau \mapsto p_{\tau(1)} \otimes \cdots \otimes p_{\tau(r)} \otimes q_{\tau(1)} \otimes \cdots \otimes q_{\tau(r)}
$$

define mutually inverse $S_{r}$-linear maps. This proves (17). Tensoring both sides over $S_{r}$ with $\operatorname{Sign}\left(S_{r}\right)$, we get (18) and therefore the proposition.

This concludes the proof of Theorem 5.1.

So we have constructed a contravariant functor

$$
\bar{K}:\{\text { Varieties }\} \rightarrow\{\text { Special } \lambda \text {-rings }\} .
$$

The Adams operations $\Psi^{n}, n \geqslant 1$, are defined on $\bar{K}(X)$ as certain universal polynomials in the $\lambda^{i}$ with integer coefficients given by (14). In particular, $\Psi^{1}=\lambda^{1}=\mathrm{id}$. We have

$$
\Psi^{n}[\mathcal{L}]=\left[\mathcal{L}^{\otimes n}\right]
$$

if $\mathcal{L}$ is a line bundle. Since the $\lambda$-ring $\bar{K}(X)$ is special, the $\Psi^{n}$ are $\lambda$-homomorphisms (see [AT69, $\S 5])$.

Finally it may be worth noting that the ordinary $K$-theory of $X$ is a quotient of $\bar{K}(X)$ in the sense of $\lambda$-rings. There is an obvious surjective homomorphism which respects $\lambda$-operations for classes of effective vector bundles and therefore for all classes by Lemma 4.4. 


\section{RATIONALITY CRITERIA FOR MOTIVIC ZETA FUNCTIONS}

\section{Motivic measures $\mu_{n}$}

Consider the $(\lambda$-)ring $\mathbb{Z}[M]$ as in Example 4.8. We will freely consider elements of the monoid $M$ either as polynomials with integer coefficients or as (isomorphism classes of) graded vector spaces.

For a smooth connected projective variety $X$ of dimension $d$ define

$$
\mu_{n}(X):=\left[1+h_{n}^{1}(X) s+\cdots+h_{n}^{d}(X) s^{d}\right] \in \mathbb{Z}[M],
$$

where

$$
h_{n}^{i}(X)=\operatorname{dim} H^{0}\left(X, \Psi^{n} \Omega_{X}^{i}\right) \in \mathbb{Z} .
$$

We constructed the measure $\mu=\mu_{1}$ in our previous paper [LL03]. Explicitly

$$
\mu_{1}(X)=\left[1+h^{1,0}(X) s+\cdots+h^{d, 0}(X) s^{d}\right]
$$

for a smooth projective irreducible $X$ of dimension $d$.

Proposition 6.1. For each $n \geqslant 1$ we have

i) $\mu_{n}(X)=\mu_{n}(\tilde{X})$ if $X$ and $\tilde{X}$ are birational,

ii) $\mu_{n}(X \times Y)=\mu_{n}(X) \mu_{n}(Y)$,

iii) $\mu_{n}\left(\mathbb{P}^{k}\right)=1$ for all $k \geqslant 0$.

Corollary 6.2. The mapping $\mu_{n}$ extends (uniquely) to a ring homomorphism

$$
\mu_{n}: K_{0}\left[\mathcal{V}_{\mathbb{C}}\right] /\left(\mathbb{A}^{1}\right) \rightarrow \mathbb{Z}[M]
$$

Indeed, this follows from [LL03, Theorem 2.3].

To prove Proposition 6.1, we need the following lemma.

Lemma 6.3. Let $X$ and $Y$ be varieties and $E$ and $F$ be vector bundles on $X$ and $Y$ respectively. Then

$$
H^{0}\left(X \times Y, p^{*} E \otimes q^{*} F\right)=H^{0}(X, E) \otimes H^{0}(Y, F) .
$$

Proof. By the projection formula $p_{*}\left(p^{*} E \otimes q^{*} F\right)=E \otimes p_{*} q^{*} F$, where $p_{*} q^{*} F$ is the trivial bundle on $X$ with fiber $H^{0}(Y, F)$. Hence

$$
\begin{aligned}
H^{0}\left(X \times Y, p^{*} E \otimes q^{*} F\right) & =H^{0}\left(X, p_{*}\left(p^{*} E \otimes q^{*} F\right)\right) \\
& =H^{0}\left(X, E \otimes p_{*} q^{*} F\right)=H^{0}(X, E) \otimes H^{0}(Y, F) .
\end{aligned}
$$

We can express Lemma 6.3 in terms of the group homomorphisms (15) by writing

$$
\operatorname{dim} H_{X \times Y}^{0}\left(\left[p^{*} E \otimes q^{*} F\right]\right)=\operatorname{dim} H_{X}^{0}([E]) \operatorname{dim} H_{Y}^{0}([F])
$$

for vector bundles $E$ and $F$ of $X$ and $Y$ respectively. The same identity extends to general classes in $\bar{K}(X)$ and $\bar{K}(Y)$ by additivity.

Proof of Proposition 6.1. i) Since $\Psi^{n}$ is a polynomial in operations $\lambda^{j}$, it suffices to prove that

$$
H^{0}\left(X, \bigotimes_{i}\left(\Omega_{X}^{i}\right)^{\otimes n_{i}}\right)=H^{0}\left(\tilde{X}, \bigotimes_{i}\left(\Omega_{\tilde{X}}^{i}\right)^{\otimes n_{i}}\right) \text {. }
$$

This follows from the well-known argument for birational invariance of geometric genus [Har77, ch. II, 8.19].

ii) Consider the projections $X \stackrel{p}{\leftarrow} X \times Y \stackrel{q}{\rightarrow} Y$. We have

$$
\Omega_{X \times Y}^{1}=p^{*} \Omega_{X}^{1} \oplus q^{*} \Omega_{Y}^{1} .
$$


Hence

$$
\Omega_{X \times Y}^{m}=\bigoplus_{i+j=m} p^{*} \Omega_{X}^{i} \otimes q^{*} \Omega_{Y}^{j}
$$

As $\bar{K}(X)$ is a special $\lambda$-ring,

$$
\begin{aligned}
\Psi^{n}\left[\Omega_{X \times Y}^{m}\right] & =\sum_{i+j=m} \Psi^{n}\left[p^{*} \Omega_{X}^{i}\right] \Psi^{n}\left[q^{*} \Omega_{Y}^{j}\right] \\
& =\sum_{i+j=m} p^{*} \Psi^{n}\left[\Omega_{X}^{i}\right] q^{*} \Psi^{n}\left[\Omega_{Y}^{j}\right]
\end{aligned}
$$

It follows that

$$
\operatorname{dim} H_{X \times Y}^{0}\left(\left[\Psi^{n} \Omega_{X \times Y}^{m}\right]\right)=\sum_{i+j=m} \operatorname{dim} H_{X}^{0}\left(\left[\Psi^{n} \Omega_{X}^{i}\right]\right) \operatorname{dim} H_{Y}^{0}\left(\left[\Psi^{n} \Omega_{Y}^{j}\right]\right),
$$

i.e. $\mu_{n}(X \times Y)=\mu_{n}(X) \mu_{n}(Y)$.

iii) Since $\mathbb{P}^{k}$ is birational to $\left(\mathbb{P}^{1}\right)^{k}$, by parts $\mathrm{i}$ and ii above it suffices to prove that $\mu_{n}\left(\mathbb{P}^{1}\right)=1$. We have

$$
\left[\Psi^{n} \Omega_{\mathbb{P}^{1}}^{1}\right]=\left[\mathcal{O}_{\mathbb{P}^{1}}(-2 n)\right],
$$

and therefore $\mu_{n}\left(\mathbb{P}^{1}\right)=[1]$. This proves the proposition.

Proposition 6.4. Let $X$ be a variety of dimension $\leqslant 2$. Then

$$
\mu_{1}\left(\operatorname{Sym}^{m} X\right)=\lambda^{m}\left(\mu_{1}(X)\right) .
$$

Proof. The class of $X$ in $K_{0}\left[\mathcal{V}_{\mathbb{C}}\right]$ is a linear combination of classes of smooth projective varieties of dimension 0, 1 and 2. By Lemma 4.4, it suffices to prove the proposition for these smooth projective varieties. Thus we may assume that $X$ is smooth projective. If $\operatorname{dim} X=0$ the assertion is trivial. We will prove the assertion in the case $\operatorname{dim} X=2$ (the case of curves is similar and simpler since $\operatorname{Sym}^{n} X$ is smooth if $\operatorname{dim} X=1$ ). So let $X$ be a smooth projective surface.

We will use the smooth variety $\operatorname{Hilb}^{m} X$, that is the Hilbert scheme of zero-dimensional subschemes of $X$. It follows immediately from the main theorem of Göttsche [Göt01] that the classes of $\mathrm{Hilb}^{m} X$ and $\operatorname{Sym}^{m} X$ are equal in $K_{0}\left[\mathcal{V}_{\mathbb{C}}\right] /\left[\mathbb{A}^{1}\right]$. In particular,

$$
\mu_{n}\left(\operatorname{Hilb}^{m} X\right)=\mu_{n}\left(\operatorname{Sym}^{m} X\right) .
$$

By Proposition 7.4 below, $H^{0}\left(\operatorname{Hilb}^{m} X, \Omega_{\text {Hilb }^{m} X}^{i}\right)=H^{0}\left(X^{m}, \Omega_{X^{m}}^{i}\right)^{S_{m}}$. Thus we need to prove the following equality in $M$ :

$$
1+H^{0}\left(X^{m}, \Omega^{1}\right)^{S_{m}} s+\cdots+H^{0}\left(X^{m}, \Omega^{2 m}\right)^{S_{m}} s^{2 m}=\lambda^{m}\left(1+H^{0}\left(X, \Omega^{1}\right) s+H^{0}\left(X, \Omega^{2}\right) s^{2}\right) .
$$

Recall from Example 4.6 that

$$
\lambda^{j}\left(V s^{i}\right)= \begin{cases}\operatorname{Sym}^{j}(V) s^{i j}, & \text { if } i \text { is even, } \\ \Lambda^{j}(V) s^{i j}, & \text { if } i \text { is odd. }\end{cases}
$$

Let $\sum_{i} V_{i} s^{i} \in M$ be a graded vector space. Then

$$
\lambda^{m}\left(\sum_{i} V_{i} s^{i}\right)=\sum_{i} \sum_{j_{1} n_{1}+\cdots+j_{k} n_{k}=i} \lambda^{n_{1}}\left(V_{j_{1}}\right) \otimes \cdots \otimes \lambda^{n_{k}}\left(V_{j_{k}}\right) s^{i} .
$$

Let us prove the following general lemma. Let $Y$ be a smooth projective variety and $F$ a vector bundle on $Y$ of rank $r$. Fix $m \geqslant 1$ and denote by $p_{i}: Y^{m} \rightarrow Y$ the projection to the $i$ th factor. Put $F_{i}:=p_{i}^{*} F, \mathcal{F}:=F_{1} \oplus \cdots \oplus F_{m}$. 


\section{RATIONALITY CRITERIA FOR MOTIVIC ZETA FUNCTIONS}

Lemma 6.5. There is a natural isomorphism of graded vector spaces

$$
\sum_{j} H^{0}\left(Y^{m}, \Lambda^{j}(\mathcal{F})\right)^{S_{m}} s^{j}=\lambda^{m}\left(\sum_{j} H^{0}\left(Y, \Lambda^{j} F\right) s^{j}\right) .
$$

Proof. We have

$$
\Lambda^{\bullet}(\mathcal{F})=\bigoplus_{\left(j_{1}, \ldots, j_{m}\right)} \Lambda^{j_{1}} F_{1} \otimes \cdots \otimes \Lambda^{j_{m}} F_{m}
$$

By Lemma 6.3,

$$
H^{0}\left(Y^{m}, \Lambda^{j_{1}} F_{1} \otimes \cdots \otimes \Lambda^{j_{m}} F_{m}\right) \simeq \bigotimes_{i=1}^{m} H^{0}\left(Y, \Lambda^{j_{i}} F\right)
$$

Put $\mathcal{F}_{j_{1} \cdots j_{m}}=\Lambda^{j_{1}} F_{1} \otimes \cdots \otimes \Lambda^{j_{m}} F_{m}$. Consider the $S_{m}$-action on the sheaf $\Lambda^{\bullet}(\mathcal{F})$. The sheaves $\mathcal{F}_{j_{1} \cdots j_{m}}$ and $\mathcal{F}_{j_{1}^{\prime} \cdots j_{m}^{\prime}}$ are in the same $S_{m}$-orbit if and only if the multisets $\left\{j_{1}, \ldots, j_{m}\right\}$ and $\left\{j_{1}^{\prime}, \ldots, j_{m}^{\prime}\right\}$ are equal. For each multiset $\left\{j_{1}, \ldots, j_{m}\right\}$ choose a representative $\left(j_{1}, \ldots, j_{m}\right)$ of the corresponding $S_{m}$-orbit. Put $G_{j_{1} \cdots j_{m}}=\operatorname{Stab}_{S_{m}}\left(j_{1}, \ldots, j_{m}\right)$. Then

$$
W=H^{0}\left(Y^{m}, \Lambda^{\bullet} \mathcal{F}\right)^{S_{m}}=\sum_{\left\{j_{1}, \ldots, j_{m}\right\}} H^{0}\left(Y^{m}, \mathcal{F}_{j_{1} \cdots j_{m}}\right)^{G_{j_{1} \cdots j_{m}}} .
$$

Consider the space

$$
H^{0}\left(Y^{m}, \mathcal{F}_{j_{1} \cdots j_{m}}\right)^{G_{j_{1} \cdots j_{m}}}=\left(\bigotimes_{s} H^{0}\left(Y^{m}, \Lambda^{j_{s}} F_{s}\right)\right)^{G_{j_{1} \cdots j_{m}}}
$$

Assume for simplicity of notation that the multiset $\left\{j_{1}, \ldots, j_{m}\right\}$ contains $k$ different elements:

$$
j_{1}=\cdots=j_{t_{1}} \neq j_{t_{1}+1}=\cdots=j_{t_{1}+t_{2}} \neq \cdots=j_{t_{1}+\cdots+t_{k}}
$$

Then $G_{j_{1} \cdots j_{m}}=S_{t_{1}} \times \cdots \times S_{t_{k}}$. For example, the subgroup $S_{t_{1}}$ acts by permuting factors in $\Lambda^{j_{1}} F_{1} \otimes$ $\cdots \otimes \Lambda^{j_{1}} F_{t_{1}}$. A transposition of two factors in this tensor product corresponds to a product of $j_{1}$ transpositions on the level of $\mathcal{F}^{\otimes j_{1} t_{1}}$. Therefore,

$$
H^{0}\left(Y^{m}, \Lambda^{j_{1}} F_{1} \otimes \cdots \otimes \Lambda^{j_{t_{1}}} F_{t_{1}}\right)^{S_{t_{1}}} \simeq \begin{cases}\operatorname{Sym}^{t_{1}} H^{0}\left(Y, \Lambda^{j_{1}} F\right), & \text { if } j_{1} \text { is even, } \\ \Lambda^{t_{1}} H^{0}\left(Y, \Lambda^{j_{1}} F\right), & \text { if } j_{1} \text { is odd. }\end{cases}
$$

The space $H^{0}\left(Y^{m}, \mathcal{F}_{j_{1} \cdots j_{m}}\right)^{G_{j_{1} \cdots j_{m}}}$ is the tensor product of $k$ factors of the form $\operatorname{Sym}^{t_{p}} H^{0}\left(Y, \Lambda^{l_{p}} F\right)$ (if $l_{p}$ is even) or $\Lambda^{t_{p}} H^{0}\left(Y, \Lambda^{l_{p}} F\right)$ (if $l_{p}$ is odd). Also the degree of this space is equal to $\sum_{s} j_{s}$. This proves the lemma.

Now apply the lemma with $Y=X$ and $F=\Omega_{X}^{1}$ to get the proposition.

Remark 6.6. Unfortunately, the assertion of Proposition 6.4 is no longer true if we replace the measure $\mu_{1}$ by $\mu_{n}$ for $n \geqslant 2$. A counter-example is provided by a smooth projective curve of genus $g \geqslant 2$. Indeed, $\operatorname{Sym}^{m} X$ is stably birational to the Jacobian of $X$ for $m \geqslant g$, and $\Omega_{\text {Jac } X}^{1}$ is trivial. It follows that

$$
\mu_{n}\left(\operatorname{Sym}^{m} X\right)=\mu_{n}(\operatorname{Jac} X)=\mu_{1}(\operatorname{Jac} X)=\mu_{1}\left(\operatorname{Sym}^{m} X\right) .
$$

On the other hand it is clear that $\mu_{n}(X)$ and hence $\lambda^{m}\left(\mu_{n}(X)\right)$ depends on $n$.

Question 6.7. Is $\mu_{1}: K_{0}\left[\mathcal{V}_{\mathbb{C}}\right] \rightarrow \mathbb{Z}[M]$ a $\lambda$-homomorphism? It seems likely to us that the answer is affirmative. We could prove it if we knew that for any smooth projective variety $Z$ and any $m \geqslant 1$ the class of $\operatorname{Sym}^{m} Z$ is equal in $K_{0}\left[\mathcal{V}_{\mathbb{C}}\right] /\left[\mathbb{A}^{1}\right]$ to the class of a resolution of $\operatorname{Sym}^{m} Z$. So one might generalize and ask the following question. Let $X$ be a non-singular complex projective variety with 


\section{LARSEN AND V. A. Lunts}

an action by a finite group $G$, and $Y$ a non-singular projective variety birationally equivalent to $X / G$. Is it always true that

$$
[X / G] \equiv[Y] \quad\left(\bmod \left[\mathbb{A}^{1}\right]\right) ?
$$

\section{Irrationality theorems}

Let $X$ be a smooth projective surface. Let $m \geqslant 1$ be an integer and Hilb $^{m} X$ be the Hilbert scheme of zero-dimensional subschemes of $X$ of length $m$. It is well known that the natural (Hilbert-Chow) morphism $g: \mathrm{Hilb}^{m} X \rightarrow \mathrm{Sym}^{m} X$ is a resolution of singularities that is a semismall map [Nak99, $1.15,6.10]$. Over the locus $U_{m} \subset \operatorname{Sym}^{m} X$ of $m$-element subsets of $X, g$ is an isomorphism, and $g^{-1}\left(U_{m}\right)$ is the open subscheme of $\mathrm{Hilb}^{m} X$ parametrizing reduced zero-dimensional subschemes of $X$ of length $m$. Note that the complement of $U_{m}$ in $\operatorname{Sym}^{m} X$ has codimension 2, but the complement of $g^{-1}\left(U_{m}\right)$ in Hilb ${ }^{m} X$ has codimension 1. It follows immediately from the main theorem of Göttsche [Göt01] that the classes of $\operatorname{Hilb}^{m} X$ and $\operatorname{Sym}^{m} X$ are equal in $K_{0}\left[\mathcal{V}_{\mathbb{C}}\right] /\left[\mathbb{A}^{1}\right]$. Thus for all $n \geqslant 1$

$$
\mu_{n}\left(\operatorname{Hilb}^{m} X\right)=\mu_{n}\left(\operatorname{Sym}^{m} X\right) .
$$

Lemma 7.1. For any $m, n \geqslant 1$ we have

$$
H^{0}\left(\operatorname{Hilb}^{m} X,\left(\Omega_{\operatorname{Hilb}^{m} X}^{1}\right)^{\otimes n}\right) \subset H^{0}\left(X^{m},\left(\Omega_{X^{m}}^{1}\right)^{\otimes n}\right)^{S_{m}} .
$$

Proof. Over $U_{m}, \pi: X^{m} \rightarrow \operatorname{Sym}^{m} X$ is étale, so

$$
\begin{aligned}
H^{0}\left(\operatorname{Hilb}^{m} X,\left(\Omega_{\mathrm{Hilb}^{m} X}^{1}\right)^{\otimes n}\right) & \subset H^{0}\left(g^{-1}\left(U_{m}\right),\left(\Omega_{g^{-1}\left(U_{m}\right)}^{1}\right)^{\otimes n}\right) \\
& =H^{0}\left(U_{m},\left(\Omega_{U_{m}}^{1}\right)^{\otimes n}\right)=H^{0}\left(\pi^{-1}\left(U_{m}\right),\left(\Omega_{\pi^{-1}\left(U_{m}\right)}^{1}\right)^{\otimes n}\right)^{S_{m}} .
\end{aligned}
$$

Moreover,

$$
H^{0}\left(\pi^{-1}\left(U_{m}\right),\left(\Omega_{\pi^{-1}\left(U_{m}\right)}^{1}\right)^{\otimes n}\right)=H^{0}\left(X^{m},\left(\Omega_{X^{m}}^{1}\right)^{\otimes n}\right),
$$

since $\pi^{-1}\left(U_{m}\right)$ has complement of codimension 2 in $X^{m}$.

Proposition 7.2. For any $m, n \geqslant 1$ we have

$$
H^{0}\left(\operatorname{Hilb}^{m} X, \omega_{\operatorname{Hilb}^{m} X}^{\otimes n}\right)=H^{0}\left(X^{m}, \omega_{X^{m}}^{\otimes n}\right)^{S_{m}} .
$$

Proof. Consider the pull-back diagram,

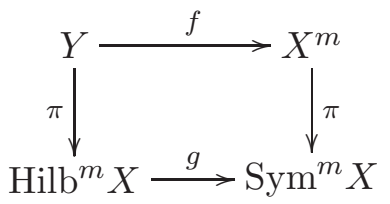

where the vertical arrows are quotient morphisms by the $S_{m}$-action. Let $\operatorname{Sym}_{\circ}^{m} X$ denote the open subspace of $\operatorname{Sym}^{m} X$ consisting of all multisets $\left\{x_{1}, \ldots, x_{m}\right\}$ in which at least $m-1$ points are distinct; it is the complement of a closed subvariety of codimension 4. Pulling back the previous diagram to $\operatorname{Sym}_{\circ}^{m} X$, we have the following diagram.

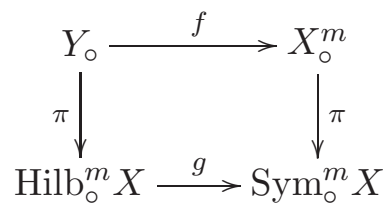

Denote by $\Delta_{\circ}$ the ramification locus of $X_{\circ}^{m}$ over $\operatorname{Sym}_{\circ}^{m} X$, that is, the closed subvariety of $X_{\circ}^{m}$ in which some pair of coordinates coincide. Then $Y_{\circ}$ is the blowup of $X_{\circ}^{m}$ along the smooth subvariety $\Delta_{\circ}$ (which is of codimension 2). So $Y_{\circ}$ is smooth. Let $E_{\circ} \subset Y_{\circ}$ be the exceptional divisor. 


\section{RATIONALITY CRITERIA FOR MOTIVIC ZETA FUNCTIONS}

The map $\pi: Y_{\circ} \rightarrow \operatorname{Hilb}_{\circ}^{m} X$ is unramified away from $E_{\circ}$ and has ramification of degree 2 along $E_{\circ}$. Since the map $g$ is semismall, the complement of the open subset Hilb ${ }_{\circ}^{m} X \subset$ Hilb $^{m} X$ has codimension $\geqslant 2$. Hence also $Y \backslash Y_{\circ}$ has codimension $\geqslant 2$ in $Y$.

It suffices to prove that

$$
H^{0}\left(\operatorname{Hilb}_{\circ}^{m} X, \omega_{\operatorname{Hilb}_{\circ}^{m} X}^{\otimes n}\right)=H^{0}\left(X_{\circ}^{m}, \omega_{X_{\circ}^{m}}^{\otimes n}\right)^{S_{m}} .
$$

We have the natural injective morphisms of coherent sheaves

$$
\alpha: \pi^{*} \omega_{\mathrm{Hilb}_{\circ}^{m} X}^{\otimes n} \rightarrow \omega_{Y_{0}}^{\otimes n}, \quad \beta: f^{*} \omega_{X_{0}^{m}}^{\otimes n} \rightarrow \omega_{Y_{\circ}}^{\otimes n} .
$$

It suffices to prove that $\operatorname{Im}(\alpha)=\operatorname{Im}(\beta)$. Indeed,

$$
H^{0}\left(Y_{\circ}, \pi^{*} \omega_{\operatorname{Hilb}_{\circ}^{m} X}^{\otimes n}\right)^{S_{m}}=H^{0}\left(\operatorname{Hilb}_{\circ}^{m} X, \omega_{\operatorname{Hilb}_{\circ}^{m} X}^{\otimes n}\right) .
$$

Both $\alpha$ and $\beta$ are surjective away from $E$. So it remains to analyze the maps $f$ and $\pi$ near $E$.

Choose a point $p \in \Delta_{\circ}$ and $q \in f^{-1}(p)$. There exist local (analytic) coordinates $x_{1}, x_{2}, \ldots, x_{2 m}$ near $p$ and local coordinates $y_{1}, \ldots, y_{2 m}$ near $q$ such that

$$
f^{*}\left(x_{1}\right)=y_{1}, f^{*}\left(x_{2}\right)=y_{1} y_{2}, f^{*}\left(x_{3}\right)=y_{3}, \ldots, f^{*}\left(x_{2 m}\right)=y_{2 m} .
$$

Then $y_{1}=0$ is the local equation of $E_{\circ}$. Thus

$$
f^{*}\left(d x_{1} \wedge \cdots \wedge d x_{2 m}\right)=y_{1} d y_{1} \wedge \cdots \wedge y_{2 m}
$$

and

$$
\beta\left(\left(d x_{1} \wedge \cdots \wedge d x_{2 m}\right)^{\otimes n}\right)=y_{1}^{n}\left(d y_{1} \wedge \cdots \wedge y_{2 m}\right)^{\otimes n},
$$

so that $\operatorname{Im}(\beta)=\omega_{Y_{*}}^{\otimes n}\left(-n E_{\circ}\right)$. Similarly, we can define local coordinates $z_{1}, \ldots, z_{2 m}$ near $\pi(q) \in$ Hilb $_{\circ}^{m} X$, so that

$$
\pi^{*}\left(z_{1}\right)=y_{1}^{2}, \pi^{*}\left(z_{2}\right)=y_{2}, \ldots, \pi^{*}\left(z_{2 m}\right)=y_{2 m} .
$$

Therefore

$$
f^{*}\left(d z_{1} \wedge \cdots \wedge d z_{2 m}\right)=2 y_{1} d y_{1} \wedge \cdots \wedge d y_{2 m}
$$

and

$$
\alpha\left(\left(d z_{1} \wedge \cdots \wedge d z_{2 m}\right)^{\otimes n}\right)=2^{n} y_{1}^{n}\left(d y_{1} \wedge \cdots \wedge y_{2 m}\right)^{\otimes n} .
$$

That means also $\operatorname{Im}(\alpha)=\omega_{Y_{*}}\left(-n E_{\circ}\right)$.

Lemma 7.3. For any $m, n \geqslant 1$ we have

$$
H^{0}\left(X^{m}, \omega_{X^{m}}^{\otimes n}\right)^{S_{m}}=\operatorname{Sym}^{m} H^{0}\left(X, \omega_{X}^{\otimes n}\right) .
$$

Proof. Let $p_{i}: X^{m} \rightarrow X$ denote the projection on the $i$ th factor. Then

$$
\omega_{X^{m}}^{\otimes n}=\left(p_{1}^{*} \omega_{X} \otimes \cdots \otimes p_{m}^{*} \omega_{X}\right)^{\otimes n} \simeq p_{1}^{*} \omega_{X}^{\otimes n} \otimes \cdots \otimes p_{m}^{*} \omega_{X}^{\otimes n},
$$

and hence

$$
H^{0}\left(X^{m}, \omega_{X^{m}}^{\otimes n}\right)=H^{0}\left(X, \omega_{X}^{\otimes n}\right)^{\otimes m} .
$$

The $S_{m}$-action permutes the factors and

$$
H^{0}\left(X^{m}, \omega_{X^{m}}^{\otimes n}\right)^{S_{m}}=\operatorname{Sym}^{m} H^{0}\left(X, \omega_{X}^{\otimes n}\right),
$$

since $\operatorname{dim} X$ is even.

Proposition 7.4. For any $m, i \geqslant 1$ we have

$$
H^{0}\left(\operatorname{Hilb}^{m} X, \Omega_{\operatorname{Hilb}^{m} X}^{i}\right)=H^{0}\left(X^{m}, \Omega_{X^{m}}^{i}\right)^{S_{m}} .
$$




\section{LARSEn And V. A. Lunts}

Proof. By that GAGA principle, we may work in the analytic category. Since $Y_{\circ}$ is a blowup of $X_{\circ}^{m}$ along a smooth subvariety, we have

$$
H^{0}\left(Y_{\circ}, \Omega_{Y_{\circ}}^{i}\right)=H^{0}\left(X_{\circ}^{m}, \Omega_{X_{\circ}^{m}}^{i}\right) .
$$

Also

$$
\begin{aligned}
H^{0}\left(X_{\circ}^{m}, \Omega_{X_{\circ}^{m}}^{i}\right) & =H^{0}\left(X^{m}, \Omega_{X^{m}}^{i}\right), \\
H^{0}\left(\operatorname{Hilb}_{\circ}^{m} X, \Omega_{\operatorname{Hilb}_{\circ}^{m} X}^{i}\right) & =H^{0}\left(\operatorname{Hilb}^{m} X, \Omega_{\operatorname{Hilb}^{m} X}^{i}\right),
\end{aligned}
$$

since $X_{\circ}^{m}$ has complement of codimension $\geqslant 2$ in $X^{m}$ and Hilb $\mathrm{b}_{\circ}^{m} X$ has complement of codimension $\geqslant 2$ in Hilb $^{m} X$. Thus we must show that

$$
H^{0}\left(\operatorname{Hilb}_{\circ}^{m} X, \Omega_{\mathrm{Hilb}_{\circ}^{m} X}^{i}\right)=H^{0}\left(Y_{\circ}, \Omega_{Y_{\circ}}^{i}\right)^{S_{m}}
$$

Since $\mathrm{Hilb}_{\circ}^{m} X=Y_{\circ} / S_{m}$ the adjunction morphism

$$
\Omega_{\mathrm{Hilb}_{\circ}^{m} X}^{i} \rightarrow \pi_{*} \pi^{-1} \Omega_{\mathrm{Hilb}}^{i}{ }_{\circ}^{m} X
$$

induces the map

$$
\Omega_{\mathrm{Hilb}_{\mathrm{o}}^{m} X}^{i} \stackrel{\sim}{\rightarrow}\left(\pi_{*} \pi^{-1} \Omega_{\mathrm{Hilb}_{\mathrm{o}}^{m} X}^{i}\right)^{S_{m}},
$$

which is an isomorphism by [Gro57, (5.1.1)]. Hence

$$
H^{0}\left(\operatorname{Hilb}_{\circ}^{m} X, \Omega_{\operatorname{Hilb}_{\circ}^{m} X}^{i}\right)=H^{0}\left(Y_{\circ}, \pi^{-1} \Omega_{\operatorname{Hilb}_{\circ}^{m} X}^{i}\right)^{S_{m}} .
$$

Every $i$-form on Hilb $\mathrm{b}_{\circ}^{m} X$ lifts by $\pi$ to an $S_{m}$-invariant $i$-form on $Y_{\circ}$. Let $\mathcal{F}$ denote the cokernel of the $S_{m}$-equivariant injective morphism

$$
\pi^{-1} \Omega_{\mathrm{Hilb}_{\circ}^{m} X}^{i} \rightarrow \Omega_{Y_{\circ}}^{i}
$$

To prove the proposition, it suffices to prove

$$
H^{0}\left(Y_{\circ}, \pi^{-1} \Omega_{\mathrm{Hilb}_{\circ}^{m} X}^{i}\right)^{S_{m}}=H^{0}\left(Y_{\circ}, \Omega_{Y_{\circ}}^{i}\right)^{S_{m}},
$$

and this follows if $H^{0}\left(Y_{\circ}, \mathcal{F}\right)^{S_{m}}=0$.

Let $q \in Y_{\circ}, G_{q}=\operatorname{Stab}(q) \subset S_{m}$. It suffices to show that $\mathcal{F}_{q}^{G_{q}}=0$ or, equivalently,

$$
\left(\pi^{-1} \Omega_{\operatorname{Hilb}_{\circ}^{m} X}^{i}\right)_{q}^{G_{q}}=\left(\Omega_{Y_{\circ}, q}^{i}\right)^{G_{q}},
$$

where (since $G_{q}$ respects $\pi$ ) the action of $G_{q}$ on the left-hand side is trivial. If $f(q)$ has distinct coordinates, then $G_{q}$ is trivial, so the map $\pi$ is étale near $q$ and $\left(\pi^{-1} \Omega_{\mathrm{Hilb}_{\circ}^{m} X}^{i}\right)_{q}=\left(\Omega_{Y_{\circ}, q}^{i}\right)$. Suppose $q \in E_{\circ}$ and hence $G_{q}=\mathbb{Z} / 2 \mathbb{Z}$. Consider local coordinates $y_{1}, \ldots, y_{2 m}$ at $q$ and local coordinates $z_{1}, \ldots, z_{2 m}$ at $\pi(q)$ as in the proof of Proposition 7.2 above, so that

$$
\pi^{*}\left(z_{1}\right)=y_{1}^{2}, \pi^{*}\left(z_{2}\right)=y_{2}, \ldots \pi^{*}\left(z_{2 m}\right)=y_{2 m} .
$$

The non-trivial element of $G_{q}$ sends $y_{1} \mapsto-y_{1}$ and $y_{i} \mapsto y_{i}$ for $i \geqslant 2$. A monomial

$$
y_{1}^{a_{1}} \cdots y_{2 m}^{a_{2 m}}\left(d y_{1}\right)^{\wedge b_{1}} \wedge \cdots \wedge\left(d y_{2 m}\right)^{\wedge b_{2 m}}
$$

(where each $b_{i} \in\{0,1\}$ ) is $G_{q}$-invariant if and only if $a_{1}+b_{1}$ is even, and in this case

$$
\begin{aligned}
y_{1}^{a_{1}} \cdots & y_{2 m}^{a_{2 m}}\left(d y_{1}\right)^{\wedge b_{1}} \wedge \cdots \wedge\left(d y_{2 m}\right)^{\wedge b_{2 m}} \\
& = \begin{cases}\pi^{*}\left(z_{1}^{a_{1} / 2} \cdots z_{2 m}^{a_{2 m}}\left(d z_{2}\right)^{\wedge b_{2}} \wedge \cdots \wedge\left(d z_{2 m}\right)^{\wedge b_{2 m}}\right) & \text { if } b_{1}=0, \\
\pi^{*}\left((1 / 2) z_{1}^{\left(a_{1}-1\right) / 2} \cdots z_{2 m}^{a_{2 m}} d z_{1} \wedge\left(d z_{2}\right)^{\wedge b_{2}} \wedge \cdots \wedge\left(d z_{2 m}\right)^{\wedge b_{2 m}}\right) & \text { if } b_{1}=1 .\end{cases}
\end{aligned}
$$

The proposition follows.

Proposition 7.5. The expression $\left|h_{n}^{i}\left(\operatorname{Hilb}^{m} X\right)\right|$ has an upper bound independent of $m$. 


\section{RATIONALITY CRITERIA FOR MOTIVIC ZETA FUNCTIONS}

Proof. Recall that $h_{n}^{i}(Z)$ is the dimension of the virtual vector space $\operatorname{dim} H^{0}\left(Z, \Psi^{n} \Omega_{Z}^{i}\right)$. The Adams operation $\Psi^{n}$ is a polynomial in the operations $\lambda^{i}$. As $\Omega_{Z}^{i}$ is a direct summand of $\left(\Omega_{Z}^{1}\right)^{\otimes i}$, every tensor product of sheaves of differentials on $Z$ is a summand of $\left(\Omega_{Z}^{1}\right)^{\otimes n}$ for some $n$. It therefore suffices to prove that, for all $n \geqslant 0$, the dimension of the space $H^{0}\left(\operatorname{Hilb}^{m} X,\left(\Omega_{\mathrm{Hilb}^{m} X}^{1}\right)^{\otimes n}\right)$ is bounded independently of $m$. By Lemma 7.1 above

$$
H^{0}\left(\operatorname{Hilb}^{m} X,\left(\Omega_{\mathrm{Hilb}^{m} X}^{1}\right)^{\otimes n}\right) \subset H^{0}\left(X^{m},\left(\Omega_{X^{m}}^{1}\right)^{\otimes n}\right)^{S_{m}} .
$$

We will prove that $\operatorname{dim} H^{0}\left(X^{m},\left(\Omega_{X^{m}}^{1}\right)^{\otimes n}\right)^{S_{m}}$ is bounded independently of $m$.

Let $p_{i}: X^{m} \rightarrow X$ be the projection to the $i$ th factor. Denote $F_{i}=p_{i}^{*} \Omega_{X}^{1}$. Then $\Omega_{X^{m}}^{1}=\bigoplus_{i=1}^{m} F_{i}$ and

$$
\left(\Omega_{X^{m}}^{1}\right)^{\otimes n}=\bigoplus_{i_{1}, \ldots, i_{n} \in\{1, \ldots, m\}} F_{i_{1}} \otimes \cdots \otimes F_{i_{n}} .
$$

The $S_{m}$-action permutes the summands $F_{i_{1}} \otimes \cdots \otimes F_{i_{n}}$ and the orbits correspond to partitions $P$ of the set $\{1, \ldots, n\}$. Thus

$$
H^{0}\left(X^{m},\left(\Omega_{X^{m}}^{1}\right)^{\otimes n}\right)^{S_{m}}=\bigoplus_{P} H^{0}\left(X^{m}, F_{P}\right)^{\operatorname{Stab}(P)}
$$

where $F_{P}$ is one of the summands in the orbit, corresponding to $P$. Fix a partition $P$. Assume for simplicity of notation that $P$ divides $\{1, \ldots, n\}$ into $k$ segments $P_{1}, \ldots, P_{k}$, where $a<b$ for each $a \in P_{s}, b \in P_{t}$ if $s<t$. Let $\alpha_{i}=\left|P_{i}\right|$. Then

$$
H^{0}\left(X^{m}, F_{P}\right)=H^{0}\left(X,\left(\Omega_{X}^{1}\right)^{\otimes \alpha_{1}}\right) \otimes \cdots \otimes H^{0}\left(X,\left(\Omega_{X}^{1}\right)^{\otimes \alpha_{k}}\right) .
$$

Therefore $\operatorname{dim} H^{0}\left(X^{m}, F_{P}\right)$ is bounded independently of $m$.

Theorem 7.6. Let $X$ be a smooth projective surface of Kodaira dimension $\geqslant 0$. Then the zeta function $\zeta_{X}(t)$ is not pointwise rational.

Proof. Let $F$ denote the field of fractions of $\mathbb{Z}[M]$. We will show that there exists $n \geqslant 1$ such that the power series

$$
1+\sum_{m=1}^{\infty} \mu_{n}\left(\operatorname{Sym}^{m} X\right) t^{m} \in F[[t]]
$$

is not rational.

First we claim that there exists $n \geqslant 1$ such that

$$
h_{n}^{2 m}\left(\operatorname{Hilb}^{m} X\right)=\operatorname{dim} H^{0}\left(\operatorname{Hilb}^{m} X, \omega_{\operatorname{Hilb}^{m} X}^{\otimes n}\right)>0
$$

for all $m \geqslant 1$. Indeed, by our assumption on $X$ there exists $n \geqslant 1$ such that $H^{0}\left(X, \omega^{\otimes n}\right) \neq 0$ and by Proposition 7.2 and Lemma 7.3,

$$
H^{0}\left(\operatorname{Hilb}^{m} X, \omega_{\operatorname{Hilb}^{m} X}^{\otimes n}\right)=\operatorname{Sym}^{m} H^{0}\left(X, \omega_{X}^{\otimes n}\right)
$$

for all $m \geqslant 1$. Fix one such $n$ and consider the motivic measure $\mu_{n}: K_{0}\left[\mathcal{V}_{\mathbb{C}}\right] \rightarrow \mathbb{Z}[M] \subset F$. We have $\mu_{n}\left(\operatorname{Sym}^{m} X\right)=\mu_{n}\left(\operatorname{Hilb}^{m} X\right)$. It follows that $\mu_{n}\left(\operatorname{Sym}^{m} X\right) \in M$ is a polynomial with constant term 1 and leading term $h_{n}^{2 d}\left(\operatorname{Hilb}^{m} X\right) s^{2 d}$.

Consider the group completion $G$ of the monoid $M$. By Lemma 4.9, $G$ is a free abelian group. Hence we may apply Theorem 2.9 to the power series $1+\sum_{m=1}^{\infty} \mu_{n}\left(\operatorname{Sym}^{m} X\right) t^{m}$. Denote $g_{m}=$ $\mu_{n}\left(\operatorname{Sym}^{m} X\right) \in M$. Assume that this power series is rational. Then there exist $k, i \geqslant 1$ and an element $g \in G$ such that

$$
g_{i+(\alpha+1) k}=g g_{i+\alpha k}
$$

for all $\alpha \geqslant 0$. The element $g$ is a rational function in $s$. Since all coefficients $g_{m}$ are non-zero polynomials in $s$, it follows that $g$ is also a non-zero polynomial. Note that $g$ is not a monomial 


\section{LARSEN AND V. A. Lunts}

since the degrees of the polynomials $g_{m}$ grow and their constant term is 1 . But then the coefficients of fixed powers of $s$ in the $g_{m}$ cannot stay bounded, which contradicts Proposition 7.5.

\section{The special Grothendieck $\lambda$-ring of varieties}

As pointed out in Example 4.10, the symmetric power operations $\mathrm{Sym}^{n}$ define a $\lambda$-ring structure on the Grothendieck ring $K_{0}\left[\mathcal{V}_{\mathbb{C}}\right]$. The zeta function $\zeta_{X}(t)$ is the universal $\lambda$-homomorphism, but it is not a ring homomorphism. To see this, it suffices to note that $\zeta_{X}(t)$ is a ratio of polynomials with constant term 1 for every curve $X$; thus (9) implies that $\zeta_{X}(t) \cdot \zeta_{Y}(t)$ is rational, while Theorem 7.6 implies that $\zeta_{X \times Y}(t)$ is irrational whenever $X$ and $Y$ both have positive genus.

Let $\left\{\lambda^{n}\right\}$ denote the $\lambda$-structure opposite to $\left\{\mathrm{Sym}^{n}\right\}$. This seems to be the natural choice of $\lambda$-structure on $K_{0}\left[\mathcal{V}_{\mathbb{C}}\right]$ insofar as $X \mapsto \operatorname{Sym}^{n} X$ behaves like a symmetric power map, for instance on cohomology. The choice does not affect which classes are virtually finite, but it will make a difference when we specialize, since, as we have seen, specialization does not commute with taking opposites.

Lemma 8.1. If $X$ is a variety over $\mathbb{C}$ whose image in $K_{0}\left[\mathcal{V}_{\mathbb{C}}\right]$ is virtually finite with respect to $\left\{\lambda_{n}\right\}$, then $\zeta_{X}(t)$ is globally rational.

Proof. Let $[X]=y-z$, where $y$ and $z$ are finite. As $\lambda_{t}$ is a ring homomorphism,

$$
\zeta_{X}(t)=\lambda_{-t}([X])^{-1}=\lambda_{-t}(y)^{-1} \lambda_{-t}(z)
$$

is globally rational in $K_{0}\left[\mathcal{V}_{\mathbb{C}}\right]$.

We have the following variant of Theorem 3.7 .

Proposition 8.2. The class of any one-dimensional variety in $K_{0}\left[\mathcal{V}_{\mathbb{C}}\right]$ is virtually finite with respect to $\left\{\lambda_{n}\right\}$.

Proof. By Corollary 3.6, $\zeta_{\mathbb{P}^{1}}(t)=(1-t)^{-1}\left(1-\left[\mathbb{A}^{1}\right] t\right)^{-1}$, so

$$
\lambda_{t}\left(\left[\mathbb{P}^{1}\right]\right)=(1+t)\left(1+\left[\mathbb{A}^{1}\right] t\right),
$$

and $\left[\mathbb{P}^{1}\right]$ is two-dimensional. By Theorem 3.7, for some positive integer $n, \zeta_{X}(t) \zeta_{\mathbb{P}^{1}}(t)^{-n}$ is a polynomial, so $n\left[\mathbb{P}^{1}\right]-[X]$ is finite-dimensional. Thus $[X]=n\left[\mathbb{P}^{1}\right]-\left(n\left[\mathbb{P}^{1}\right]-[X]\right)$ is a difference of finite-dimensional classes.

Corollary 8.3. The virtual finiteness of a complex surface $X$ depends only on the birational class of $X$.

Definition 8.4. We call the specialization of the Grothendieck ring $K_{0}\left[\mathcal{V}_{K}\right]$ with respect to $\lambda^{n}$ the Grothendieck $\lambda$-ring of $K$ and denote it $K_{0}\left[\mathcal{V}_{K}\right]_{\sigma}$. The image of $\zeta_{X}(t)$ in $K_{0}\left[\mathcal{V}_{K}\right]_{\sigma}$ will be denoted $\zeta_{X \sigma}(t)$.

In any special $\lambda$-ring, the set of virtually finite elements is clearly a $\lambda$-subring. In particular, we have the following proposition.

Proposition 8.5. For every $X / \mathbb{C}$ that is virtually finite in $K_{0}\left[\mathcal{V}_{\mathbb{C}}\right]_{\sigma}$ and every positive integer $n$, $\mathrm{Sym}^{n} X$ is again virtually finite in $K_{0}\left[\mathcal{V}_{\mathbb{C}}\right]_{\sigma}$.

Proof. The identities relating symmetric and exterior powers show that $\operatorname{Sym}^{n} X$ lies in the $\lambda$-subring of $K_{0}\left[\mathcal{V}_{\mathbb{C}}\right]_{\sigma}$ generated by $X$.

Proposition 8.6. Every principally polarized abelian surface $X / \mathbb{C}$ is virtually finite in $K_{0}\left[\mathcal{V}_{\mathbb{C}}\right]_{\sigma}$. 


\section{RATIONALITY CRITERIA FOR MOTIVIC ZETA FUNCTIONS}

Proof. It is well known that every principally polarized abelian variety of dimension $\leqslant 2$ is a product of Jacobian varieties. (In fact, this is true also in dimension 3, but we do not need it. We do not know who first made this observation, but it is an immediate consequence of Torelli's theorem and Baily's theorem [Bai60].) It suffices, therefore, to prove that the Jacobian $J$ of a genus 2 curve $X$ is virtually finite. The map $\operatorname{Sym}^{2} X \rightarrow J$ is a birational equivalence, so the proposition follows from Corollary 8.3 and Proposition 8.5.

We remark that the fact that sufficiently high symmetric powers of a non-singular projective curve are projective space bundles over its Jacobian variety does not immediately imply virtual finiteness of Jacobians, since it is not obvious that the virtual finiteness of a projective space bundle or even a vector bundle over a given variety implies the virtual finiteness of that variety. However, it is easy to prove the pointwise rationality of $\zeta_{J \sigma}(t)$ for Jacobians $J$.

Question 8.7. Is $\zeta_{A \sigma}(t)$ rational (globally or pointwise) for all abelian varieties $A$ ?

More optimistically, we might ask the following.

Question 8.8. Is $\zeta_{X \sigma}(t)$ rational for all varieties $X$ ? Is the Grothendieck $\lambda$-ring of $\mathbb{C}$ finite-dimensional in the sense of Definition 4.5?

\section{ACKnowledgements}

We would like to thank the referee for calling our attention to many obscurities and several inaccuracies in earlier versions of this paper and for making a number of helpful suggestions regarding exposition.

\section{REFERENCES}

AT69 M. Atiyah and D. Tall, Group representations, $\lambda$-rings, and the J-homomorphism, Topology 8 (1969), 253-297.

Bai60 W. Baily, On the moduli of Jacobian varieties, Ann. of Math. (2) 71 (1960), 303-314.

BGI77 P. Berthelot, A. Grothendieck and L. Illusie, Théorie des intersections et théorème de RiemannRoch, Lecture Notes in Mathematics, vol. 225 (Springer, Berlin, 1977).

BKL76 S. Bloch, A. Kas and D. Lieberman, Zero cycles on surfaces with $p_{g}=0$, Compositio Math. 33 (1976), 135-145.

Blo75 S. Bloch, $K^{2}$ of Artinian $\mathbb{Q}$-algebras, with application to algebraic cycles, Comm. Algebra 3 (1975), 405-428.

Bor94 E. Borel, Sur une application d'un théorème de M. Hadamard, Bull. Sci. Math., Sér. 218 (1894), $22-25$.

Göt01 L. Göttsche, On the motive of the Hilbert scheme of points on a complex surface, Math. Res. Lett. 8 (2001), 613-627.

Gro57 A. Grothendieck, Sur quelques points d'algèbre homologique, Tôhoku Math. J. 9 (1957), 119-221.

Har77 R. Hartshorne, Algebraic geometry (Springer, New York, 1977).

Kap00 M. Kapranov, The elliptic curve in the S-duality theory and Eisenstein series for Kac-Moody groups, Preprint (2000), math.AG/0001005.

Knu73 D. Knutson, $\lambda$-rings and the representation theory of the symmetric group, Lecture Notes in Mathematics, vol. 308 (Springer, Berlin, 1973).

LL03 M. Larsen and V. Lunts, Motivic measures and stable birational geometry, Mosc. Math. J. 3 (2003), 85-95.

Mac95 I. G. Macdonald, Symmetric functions and Hall polynomials, second edition (Clarendon Press, Oxford, 1995). 


\section{RATIONALITY CRITERIA FOR MOTIVIC ZETA FUNCTIONS}

Mum68 D. Mumford, Rational equivalence of 0-cycles on surfaces, J. Math. Kyoto Univ. 9 (1968), 195-204.

Nak99 H. Nakajima, Lectures on Hilbert schemes of points on surfaces, Amer. Math. Soc. Univ. Lect. Ser., vol. 18 (American Mathematical Society, Providence, RI, 1999).

Poo02 B. Poonen, The Grothendieck ring of varieties is not a domain, Math. Res. Lett. 9 (2002), 493-498.

Rei97 M. Reid, Chapters on algebraic surfaces, in Complex algebraic geometry, ed. J. Kollár (American Mathematical Society, Providence, RI, 1997).

Michael Larsen larsen@math.indiana.edu

Department of Mathematics, Indiana University, Bloomington, IN 47405, USA

Valery A. Lunts vlunts@indiana.edu

Department of Mathematics, Indiana University, Bloomington, IN 47405, USA 